\title{
Chord Uniqueness and Controllability: the View from the Boundary, I
}

\author{
Robert Gulliver and Walter Littman
}

December 21, 2000

\author{
School of Mathematics \\ UNIVERSITY OF MiNNESOTA \\ MinneAPolis, Minnesota 55455, USA \\ gulliver@math.umn.edu, littman@math.umn.edu
}

\begin{abstract}
Consider the problem of a compact, $n$-dimensional Riemannian manifoldwith-boundary $\bar{\Omega}$ and the natural hyperbolic P.D.E. (Riemannian wave equation):

$$
\frac{\partial^{2} u}{\partial t^{2}}=\Delta_{g} u
$$

plus possible lower-order terms, where $\Delta_{g}$ is the Riemannian Laplace operator, or Laplace-Beltrami operator, of $\Omega$. We consider the problem of the control in time $T$ of the wave equation from the boundary $\partial \Omega$ of $\Omega$, by specifying Dirichlet boundary controls on $\partial \Omega \times[0, T]$. The question we address is whether, for any Cauchy data on $\Omega$ at the initial time $t=0$, there is a choice of boundary control which will achieve any prescribed Cauchy data at the terminal time $t=T$.

In order to reduce this controllability question to a computable problem about geodesics on $\Omega$, we pose the question: are chords unique? Here, a chord is a length-minimizing geodesic of $\bar{\Omega}$ joining two given points of $\partial \Omega$. We assume that any two points of $\partial \Omega$ are connected by at most one (and hence exactly one) chord.

If, in addition, the chords are nondegenerate and $\partial \Omega$ has positive second fundamental form, then the wave equation is controllable from $\partial \Omega$ in any time $T$ greater than the maximum distance in $\bar{\Omega}$ between points of $\partial \Omega$.

This result provides a counterpoint to controllability theorems such as those in [14], [7] and [8], in which the existence of a convex function, and henceroughly speaking - an upper bound on sectional curvature, is assumed. We require no direct hypothesis on the Riemannian metric in the interior of $\Omega$.
\end{abstract}




\section{Introduction}

Whenever one approaches a subject from two different directions, there is bound to be an interesting theorem explaining their relation.

Robert Hartshorne, Notices Amer. Math. Soc. April, 2000, p. 464.

Although the subject of boundary control of partial differential equations is about a quarter of a century old, and that of Riemannian geometry much older still, there has been relatively little interaction between the two. This is especially surprising in view of the rôle bicharacteristics play in boundary control, which naturally bring to mind geodesics - a basic concept in Riemannian geometry.

One of the raisons d'être of this conference is the belief that both subjects have much to gain by closer interaction with one other. We hope that this paper makes a - perhaps modest - contribution in that direction. To be more specific, we believe there is a reservoir of as yet untapped Riemannian-geometric tools which could be applied successfully in boundary control. As one example, we cite Theorem 2 of [6], which is crucial to the proof of Proposition 5.1 below. On the other side, we expect that some of the compelling problems of the theory and applications of boundary control will stimulate the disciplines of Riemannian and Lorentzian geometry to undertake new areas of research.

Since our focus in this paper is on the relationship between Riemannian geometry and boundary control, we shall not attempt here to express controllability in terms of the optimal choice of Sobolev spaces, leaving such questions to other papers such as [9]; nor shall we attempt to find the optimal smoothness of the Riemannian metric and of other coefficients of the hyperbolic equation (see Michael Taylor's forthcoming book [16].)

Consider a compact, $n$-dimensional Riemannian manifold-with-boundary $\bar{\Omega}$. We assume that $\partial \Omega$ is smooth and nonempty, and that the metric of $\bar{\Omega}$ is smooth, i.e., $C^{\infty}$. We are interested in the boundary control of the following natural hyperbolic partial differential equation (Riemannian wave equation) on $\Omega \times[0, T]$ :

$$
\frac{\partial^{2} u}{\partial t^{2}}=\Delta_{g} u:=\sum_{i, j=1}^{n} \frac{1}{\gamma} \frac{\partial}{\partial x_{i}}\left(\gamma g^{i j}(x) \frac{\partial u}{\partial x_{j}}\right)
$$

for all $(x, t) \in \Omega \times[0, T]$, where $\left(x_{1}, \ldots, x_{n}\right)$ are arbitrary local coordinates, $g^{i j}(x)$ are the entries of the inverse matrix to the coefficients $g_{i j}(x)$ of the Riemannian metric, and $\gamma$ is the Riemannian volume integrand: $\gamma(x)=\sqrt{\operatorname{det}\left(g_{i j}(x)\right)}$. We consider the problem of the control in time $T$ of equation (1.1) from the boundary $\partial \Omega$. More 
precisely, we consider the boundary conditions

$$
u(x, t)=U(x, t) \text { for all }(x, t) \text { on } \partial \Omega \times[0, T],
$$

where $U \in H^{1 / 2}(\partial \Omega \times[0, T])$ is the control, i.e. a function which may be chosen as needed. The controllability question is whether, given any initial conditions

$$
u(x, 0)=u_{0}(x), \quad \frac{\partial u}{\partial t}(x, 0)=u_{1}(x),
$$

with finite energy, there is a choice of controls $U \in H^{1 / 2}(\partial \Omega \times[0, T])$ such that the solution of (1.1) with initial conditions (1.3) and boundary conditions (1.2) vanishes identically on $\Omega \times[T, \infty)$. Equivalently, we ask whether for some choice of controls $U$ the terminal Cauchy values vanish: $u(x, T)=0, \frac{\partial u}{\partial t}(x, T)=0$ for all $x$ in $\Omega$.

More generally, we shall consider the hyperbolic equation with additional lowerorder terms:

$$
\frac{\partial^{2} u}{\partial t^{2}}=\Delta_{g} u+\sum_{i=1}^{n} V^{i}(x, t) \frac{\partial u}{\partial x_{i}}+a(x, t) u
$$

where $\left(V^{1}, \ldots, V^{n}\right)$ are the components, in any local system of coordinates $x=$ $\left(x_{1}, \ldots, x_{n}\right)$ for $\bar{\Omega}$, of a vector field $V$ on $\bar{\Omega}$, and $a: \bar{\Omega} \rightarrow \mathbb{R}$. The first-order term $\sum_{i=1}^{n} V^{i}(x, t) \frac{\partial u}{\partial x_{i}}$ is invariant under change of coordinates. We shall assume throughout that $V(x, t)$ and $a(x, t)$ describe real-analytic mappings from $t$ to the space of smooth vector fields and smooth functions, resp., on $\bar{\Omega}$.

In section 3 below, we shall show how this control problem is related to a problem about geodesics of $\Omega$, namely, their maximum length before leaving $\Omega$. In section 4 below, we shall see how this geometric problem is related to the question whether chords are unique. Here, a chord is defined to be a length-minimizing curve in $\bar{\Omega}$ joining two given points of $\partial \Omega$. The existence of a chord joining any two points of $\partial \Omega$ is well known, although in general it may have nongeodesic segments lying in $\partial \Omega$ (see e.g. pp. 147-148 of [4].) If, however, we assume that $\partial \Omega$ is strictly convex, that is, has positive second fundamental form, then for any length-minimizing curve $\sigma:\left[s_{0}, s_{1}\right] \rightarrow \bar{\Omega}$ between two points of $\bar{\Omega}$, we have $\sigma\left(\left(s_{0}, s_{1}\right)\right) \subset \Omega$ (see the proof of Corollary 3.3 or Lemma 4.1 below.) In particular, every chord of $\bar{\Omega}$ is a geodesic in this case. We say that a chord $\sigma:\left[s_{0}, s_{1}\right] \rightarrow \bar{\Omega}$ is nondegenerate if there are no conjugate points to $\sigma\left(s_{0}\right)$ along $\sigma\left(\left(s_{0}, s_{1}\right]\right)$ (see Subsection 4.2 below.)

Another way of viewing these hypotheses of chord uniqueness and nondegeneracy is as follows. Consider any two boundary points $p$ and $q$. Suppose that, among all the light rays leaving $q$ simultaneously and propagating in $\Omega$ (without reflection), only one ray reaches $p$ first. This is equivalent to the uniqueness of chords. Nondegeneracy of chords is equivalent to the statement that, in the situation just 
described, when a ray leaving $q$ turns out to be a chord from $q$ to $p$, the boundary point $p$ depends in a diffeomorphic way on the initial direction of the ray at $q$ (see also the proof of Proposition 4.6.)

Theorem 1.1 Suppose that any two boundary points of the manifold $\bar{\Omega}$ are connected by a unique chord, which is nondegenerate. Assume that $\partial \Omega$ has positive second fundamental form. Then the hyperbolic equation (1.4) is controllable from $\partial \Omega$ in the sense of boundary conditions (1.2) in any time

$$
T>T_{0}:=\operatorname{diam}_{\bar{\Omega}}(\partial \Omega)
$$

Here, the diameter of the boundary of $\Omega$ is the maximum distance between any two of its points, with respect to the distance measured in $\bar{\Omega}$, that is: the length of the longest chord of $\Omega$. Our convention for the sign of the second fundamental form is such that if $\Omega$ is a ball of radius $r$ in $\mathbb{R}^{n}$, with the Euclidean metric, then $\partial \Omega$ has positive second fundamental form $B=r^{-1} d s^{2}$.

The "uniqueness" of a chord $\gamma:[0, a] \rightarrow \bar{\Omega}$ is understood modulo reparameterizations $s \mapsto \gamma(A s+B)(A, B \in \mathbb{R})$ of the independent variable $s$.

We shall refer to the infimal value $T_{0}$ as the "optimal time of control," even though $T_{0}$ itself may not be a control time.

As the reader will verify immediately, Theorem 1.1 follows from Proposition 3.1, Proposition 3.2 and Theorem 4.5 below. For the main geometric result of this paper, some readers will prefer the statement of Theorem 4.5 over Theorem 1.1.

Remark 1 A sufficient condition for the uniqueness of chords, and for their nondegeneracy, is that for any point $q \in \partial \Omega$, the gradient of the distance function $d_{\bar{\Omega}}(\cdot, q)$ is a continuous function of $p \in \partial \Omega, p \neq q$. In this case, the optimal time of control $T_{0}$ is the maximum of $d_{\bar{\Omega}}(p, q)$ over $p, q \in \partial \Omega$. No further information about the coefficients in the interior of $\Omega$ is required. See Proposition 4.6 below.

If, on the other hand, information is available giving upper bounds on sectional curvatures in various regions of $\Omega$, then Corollary 5.2 below provides a useful criterion for the time of boundary controllability.

Another sufficient condition for chord uniqueness is that every geodesic of $\bar{\Omega}$ starting from $\partial \Omega$ will leave $\bar{\Omega}$ before any conjugate point appears. See Proposition 4.7 below.

In Section 2 below, we shall indicate how geometric methods may be introduced for the analysis of a partial differential equation in $\mathbb{R}^{n}$ with coefficients depending on the space variables. 
In Section 4, we shall first outline some of the known concepts and results of Riemannian geometry which will be relevant to the proof of our theorems. We shall then introduce the main tools, including especially the chord map, to prove a new geometric result (Theorem 4.5), which will be a key step towards the proof of Theorem 1.1.

In Section 5, we shall present five explicit and, we hope, interesting examples to illuminate the variety of Riemannian manifolds in terms of the existence of a convex function; the uniqueness of chords; the nondegeneracy of certain geodesics; and continuity, as opposed to smoothness, of the gradient of the distance function. We shall also give criteria for the absence of conjugate points (Proposition 5.1), necessary conditions for the existence of a convex function (Proposition 5.3), and necessary conditions for chord uniqueness (Proposition 5.4.)

In part II of this paper, we plan to treat the case where control occurs only on a prescribed open subset of $\partial \Omega$.

The first author would like to acknowledge partial research support by the Max Planck Institute for Mathematics in the Sciences, Leipzig, Germany, by the University of Melbourne, Australia and by Monash University, Australia.

\section{Geometry and Partial Differential Equations}

This section and Section 4.1 below are of a tutorial character, and will outline some well-known concepts and results of differential geometry. Specifically, we shall illustrate here how Riemannian geometry can be introduced to aid in understanding properties of a hyperbolic P.D.E. In the reverse direction, this framework may be applied to any Riemannian manifold $\bar{\Omega}$ which is diffeomorphic to the closure of a smooth domain in $\mathbb{R}^{n}$.

Consider a hyperbolic equation with time-independent coefficients in a smooth domain $\Omega \subset \mathbb{R}^{n}$ :

$$
\frac{\partial^{2} u}{\partial t^{2}}=\frac{\partial}{\partial x_{i}}\left(g^{i j}(x) \frac{\partial u}{\partial x_{j}}\right)+\text { lower order terms }
$$

(the summation over $1 \leq i, j, k \leq n$ is implicit where the index appears twice in one term.) For each $x \in \bar{\Omega}$, let $g_{i j}(x)$ be the inverse matrix of $g^{i j}(x)$. Then the metric $d s^{2}=g_{i j}(x) d x_{i} d x_{j}$ makes $\bar{\Omega}$ into a Riemannian manifold-with-boundary (see [4].) After adding appropriate lower-order terms, (2.1) becomes the Riemannian wave equation (1.1) above. Observe that, since equations (1.1), (1.4) and (2.1) have the same bicharacteristics, they will also have the same controllability properties (cf. Proposition 3.2 below.) 
The length of a curve in $\bar{\Omega}$ is the integral of $d s$, and a geodesic is a curve $\sigma=$ $\left(\sigma_{1}, \ldots, \sigma_{n}\right):(a, b) \rightarrow \bar{\Omega}$ which satisfies the equation

$$
\frac{d^{2} \sigma_{k}}{d s^{2}}+\Gamma_{i j}^{k} \frac{d \sigma_{i}}{d s} \frac{d \sigma_{j}}{d s}=0 .
$$

Here $\Gamma_{i j}^{k}=\Gamma_{i j}^{k}(x)$ are the Christoffel symbols, evaluated at $x=\sigma(s)$. The Christoffel symbols are the coefficients of the Levi-Civita connection of $\bar{\Omega}$, determined by

$$
2 g_{k l} \Gamma_{i j}^{l}=\frac{\partial g_{i k}}{\partial x_{j}}-\frac{\partial g_{i j}}{\partial x_{k}}+\frac{\partial g_{k j}}{\partial x_{i}}
$$

Given a vector field $J=J^{i}(s) \frac{\partial}{\partial x_{i}}(\sigma(s))$ along $\sigma$, the covariant derivative $J^{\prime}(s)=\frac{D J}{d s}$ of $J$ with respect to $s$ may be written in terms of the Christoffel symbols:

$$
J^{\prime}(s)=\left[\frac{d J^{k}}{d s}+\Gamma_{i j}^{k}(\sigma(s)) J^{i}(s) \frac{d \sigma_{j}}{d s}(s)\right] \frac{\partial}{\partial x_{k}}(\sigma(s)),
$$

which is independent of the choice of coordinates. With this notation, the geodesic equation becomes $\sigma_{0}^{\prime \prime}(s)=0$.

The hypothesis of Theorem 1.1 that $\partial \Omega$ have positive second fundamental form at each point $p \in \partial \Omega$ may be computed most easily by making a linear change of coordinates so that the coordinate hyperplane $x_{n}=$ const. which passes through $p$ is tangent to $\partial \Omega$ there, and so that the $n$th coordinate vector is the inward unit normal to $\partial \Omega$ at $p$. Let $\partial \Omega$ be represented locally as the graph $x_{n}=f\left(x_{1}, \ldots, x_{n-1}\right)$. Then one requires that the symmetric matrix with entries

$$
B_{i j}=\Gamma_{i j}^{n}+\frac{\partial f}{\partial x_{i}} \Gamma_{n j}^{n}+\frac{\partial f}{\partial x_{j}} \Gamma_{i n}^{n}+\frac{\partial f}{\partial x_{i}} \frac{\partial f}{\partial x_{j}} \Gamma_{n n}^{n},
$$

$1 \leq i, j \leq n-1$, evaluated at $p$, be positive definite. The matrix $\left(B_{i j}\right)$ represents the second fundamental form of $\partial \Omega$ in these coordinates. Equivalently, if $\bar{\Omega}$ is extended to be a smooth subdomain of a Riemannian manifold $M$, one requires that any geodesic of $M$ which is tangent to $\partial \Omega$ at $p$ remain outside of $\bar{\Omega}$ to second order at $p$. This property has been called "pseudo-convexity" in the P.D.E. literature; in this paper, since we deal only with quantities which are invariant under smooth changes of coordinates, the convexity of a domain with respect to the affine structure of $\mathbb{R}^{n}$ will not be relevant.

\section{Bicharacteristics and Controllability}

In this section, we shall indicate the relationship between the geodesics of the Riemannian manifold $\Omega$ and the boundary controllability of equation (1.1), equation (1.4) or of equation (2.1) above. 
For the two propositions below, we assume that $\bar{\Omega}$ is a compact $n$-dimensional Riemannian manifold-with-boundary. Let $\bar{\Omega}$ be extended to become a subset of an open $n$-dimensional Riemannian manifold $M$.

Proposition 3.1 Let $M$ be a Riemannian manifold. Then the bicharacteristics of equation (1.4) are the graphs in $M \times \mathbb{R}$ of geodesics of $M$, with unit-speed parameter identified with time $\in \mathbb{R}$.

Proof. See [5], p. 209.

Q.E.D.

Proposition 3.2 If every bicharacteristic in $\bar{\Omega} \times(0, T)$ enters or leaves $\bar{\Omega} \times(0, T)$ across the lateral boundary $\partial \Omega \times(0, T)$, then boundary control is available in any time $\geq T$. Conversely, if there is a single bicharacteristic in $\Omega \times(0, T)$ that enters $\bar{\Omega} \times[0, T]$ through the open bottom $\Omega \times\{0\}$ and leaves through $\Omega \times\{T\}$, without hitting the lateral boundary, then boundary control in time $T$ is not possible.

Proof. See [11], where the proof, which is given for a bounded domain in $\mathbb{R}^{n}$, carries over without difficulty to manifolds. The assumption made in [11] that the coefficients be real-analytic is easily removed in the case of time-independent $C^{\infty}$ coefficients. See for example [1], p. 1050, where a proof is given (in $\mathbb{R}^{n}$ ) with optimal Sobolev spaces. The proof of [11] also works if the lower-order terms (i.e. not in the principal part) are real-analytic mappings from $t$ to the space of $C^{\infty}$ vector fields or $C^{\infty}$ functions on $\bar{\Omega}$. The proof should then be supplemented by uniqueness theorem 2' of [12]. Recent results in propagation of singularities by M. Taylor (see [16]) further indicate that the required smoothness in $x$ of the coefficients can be reduced to $C^{2, \alpha}$.

The converse follows from the propagation of singularities (see [13].) Namely, initial data can be constructed which is not $C^{\infty}$ near a point inside $\Omega$ such that this singularity is propagated along the bicharacteristic, without being affected by boundary values, to form a singularity at time $T$ inside $\Omega$. Thus the solution cannot have terminal Cauchy conditions $u(\cdot, T) \equiv 0, \frac{\partial u}{\partial t}(\cdot, T) \equiv 0 . \quad$ Q.E.D.

Remark 2 The reader might note, in particular, that if $\Omega$ contains a closed geodesic, then boundary control is impossible in any finite time.

Remark 3 It will be observed that if the hypothesis of the first part of Proposition 3.2 holds for a Riemannian manifold $\bar{\Omega}$, then it also holds for any compact subdomain $\bar{\Omega}_{1} \subset \Omega$. 
Corollary 3.3 If $\partial \Omega$ has positive second fundamental form, and if $\bar{\Omega}$ is not simply connected, then equation (1.1) is not controllable from the boundary in any finite time.

Proof. Let $\gamma_{1}$ be a non-contractible closed curve in $\bar{\Omega}$. The infimum of lengths of curves in the homotopy class of $\gamma_{1}$ in $\bar{\Omega}$ is assumed by a closed curve $\gamma_{0}$. Since the second fundamental form of $\partial \Omega$ is positive, $\gamma_{0}$ lies entirely in the interior $\Omega$. Otherwise, pushing off locally from $\partial \Omega$, with distance equal to a smooth nonnegative function of small support on $\partial \Omega$, would decrease length strictly. Therefore, $\gamma_{0}$ is a closed geodesic. The conclusion now follows from Remark 2 .

Q.E.D.

\section{Chord Uniqueness and Identification of Geodesics}

\subsection{Geodesics and Jacobi Fields in a Riemannian Manifold}

Let $\bar{\Omega}$ be a smooth, compact Riemannian manifold-with-boundary, of dimension $n$, and write $\langle X, Y\rangle$ for the Riemannian metric applied to tangent vectors $X, Y$ at a point of $\bar{\Omega}$. A curve $\sigma:[0, a] \rightarrow \bar{\Omega}$ is a geodesic if its tangent vector $\sigma^{\prime}(s)$ has vanishing covariant derivative along $[0, a]$ (see equation (2.2) above.) A curve with constant speed and of shortest length among curves joining its endpoints is a geodesic, as long as it remains in the open manifold $\Omega$, although not all geodesics have shortest length, even when they lie entirely in the open manifold $\Omega$. Given $p, q \in \bar{\Omega}$, write $d(p, q)$ for the infimum of lengths of curves in $\bar{\Omega}$ joining $p$ to $q$. Since $\bar{\Omega}$ is compact, and assuming that $\partial \Omega$ has positive second fundamental form, any two points $p, q$ of $\bar{\Omega}$ may be joined by a geodesic of minimum length $d(p, q)$ (see [4], pp. 147-148.)

Where convenient, and without loss of generality, we shall assume that $\bar{\Omega}$ is a compact subset of an open Riemannian manifold $M$, from which it inherits its Riemannian metric. In particular, at each point $x \in \bar{\Omega}$, the tangent space $T_{x}(\bar{\Omega})$ will be isomorphic to the vector space $\mathbb{R}^{n}$.

Let $\sigma_{0}:\left[0, a_{0}\right] \rightarrow \bar{\Omega}$ be a geodesic. Consider the linearized geodesic equation, or $J a c o b i$ equation, for a vector field $J(s)$ along $\sigma_{0}$ :

$$
J^{\prime \prime}(s)+R\left(\sigma_{0}^{\prime}(s), J(s)\right) \sigma_{0}^{\prime}(s)=0 .
$$

Here, $R(\cdot, \cdot) \cdot$ is the curvature tensor of the Riemannian manifold $\bar{\Omega}$. For example, if $\bar{\Omega}$ is an open subset of the $n$-dimensional sphere of radius $r$ in $\mathbb{R}^{n+1}$, then $R\left(\sigma^{\prime}, J\right) \tau^{\prime}=$ $r^{-2}\left[<\sigma^{\prime}, \tau^{\prime}>J-<J, \tau^{\prime}>\sigma^{\prime}\right]$ (see [4], p. 89.) $J^{\prime}(s)$ and $J^{\prime \prime}(s)$ are the first and second covariant derivatives of $J(s)$ with respect to $s$ along $\sigma_{0}$. A solution to (4.1) is called a Jacobi field. 
We begin by recalling three well-known facts about the Jacobi equation (see e.g. [4].)

First, let $\left\{\sigma_{\lambda}:-\varepsilon<\lambda<\varepsilon\right\}$ be a one-parameter family of geodesics $\sigma_{\lambda}:\left[0, a_{\lambda}\right] \rightarrow$ $\Omega$, each parameterized with constant speed. Then the transverse vector field $J(s)=$ $\left.\frac{\partial \sigma_{\lambda}(s)}{\partial \lambda}\right|_{\lambda=0}$ is a Jacobi field. Conversely, any Jacobi field along $\sigma_{0}$ arises as the transverse vector field to some family $\left\{\sigma_{\lambda}:-\varepsilon<\lambda<\varepsilon\right\}$ of geodesics.

Next, if $\sigma_{0}:\left[0, a_{0}\right] \rightarrow \bar{\Omega}$ minimizes the length between its endpoints, then any Jacobi field $J(s)$ along $\sigma_{0}$ with the initial condition $J(0)=0$ will remain nonzero for $0<s<a_{0}$. This is Jacobi's theorem; see [4], p. 248. As a partial converse, if every Jacobi field along $\sigma_{0}$ with the initial value zero remains nonzero on $\left(0, a_{0}\right]$, then $\sigma_{0}$ has minimum length among curves connecting $\sigma_{0}(0)$ with $\sigma_{0}\left(a_{0}\right)$ and lying in a sufficiently small neighborhood of $\sigma_{0}\left(\left[0, a_{0}\right]\right)$. This is proved by invoking the Gauss Lemma and constructing a field of extremals; see [4], p. 71.

Last, a Jacobi field with initial inner products $\left\langle J(0), \sigma_{0}^{\prime}(0)\right\rangle=\left\langle J^{\prime}(0), \sigma_{0}^{\prime}(0)\right\rangle$ $=0$ will remain orthogonal to the tangent vector $\sigma_{0}^{\prime}(s)$ on $0 \leq s \leq a_{0}$.

We define the sphere bundle $S(\bar{\Omega})$ of $\bar{\Omega}$ as the set of all $(x, w)$ where $x \in \bar{\Omega}$ and $w$ is a unit tangent vector to $\bar{\Omega}$ at $x$. Since the value of the constant speed of a geodesic $\sigma$ will be unimportant, it will be convenient to use initial conditions $\left(\sigma(0), \sigma^{\prime}(0)\right)=(x, w) \in S(\bar{\Omega})$ for the geodesic equation. Then convergence of the initial values $\in S(\bar{\Omega})$ is equivalent to convergence in $C^{\infty}\left(\left[0, a_{0}\right]\right)$ of the unit-speed geodesics. In the context of the sphere bundle, $\pi: S(\bar{\Omega}) \rightarrow \bar{\Omega}$ will denote the natural projection $\pi(x, w):=x$.

For $x \in \bar{\Omega}$, the exponential map $\exp _{x}: T_{x} \bar{\Omega} \rightarrow \bar{\Omega}$ is defined on a star-shaped subset of the tangent space $T_{x} \Omega$ so that for $(x, w) \in S(\bar{\Omega})$ and $t \geq 0, t \mapsto \exp _{x}(t w)=$ $\gamma(t)$ is the geodesic with initial conditions $\gamma(0)=x, \gamma^{\prime}(0)=w$.

The distance to the cut point $c: S(\bar{\Omega}) \rightarrow(0, \infty]$ is defined by

$$
c(x, w):=\sup \left\{t: \exp _{x}(t w) \text { is defined and } d\left(x, \exp _{x}(t w)\right)=t\right\}
$$

According to the proof of Theorem 3.1 of [2], the function $c: S(\bar{\Omega}) \rightarrow(0, \infty)$ is continuous provided that $\bar{\Omega}$ is compact and that any minimizing geodesic from a point of $\bar{\Omega}$ to a boundary point $q$ is transversal to $\partial \Omega$ at $q$. We may define a star-shaped open set $\mathcal{E}_{x} \subset T_{x} \Omega$ as $\left\{t w \in T_{x} \Omega:|w|=1,0 \leq t<c(x, w)\right\}$, and write $\mathcal{W}_{x} \subset \bar{\Omega}$ for its image under $\exp _{x}$. Then $\partial \mathcal{W}_{x}=\exp _{x}\left(\partial \mathcal{E}_{x}\right)$ is called the cut locus of $x$. Note that $\exp _{x}$ maps $\mathcal{E}_{x}$ diffeomorphically onto $\mathcal{W}_{x}$. A cut point $y \in \partial \mathcal{W}_{x}$ is either a boundary point of $\Omega$; the first conjugate point along a length-minimizing geodesic from $x$ (see section 4.2 below); or the end point of two distinct length-minimizing geodesics from $x$. 


\subsection{Conjugate points}

Two points $\gamma\left(s_{1}\right)$ and $\gamma\left(s_{2}\right)$ of a geodesic $\gamma$ are called conjugate points if $s_{1} \neq s_{2}$ and there exists a nontrivial Jacobi field $J$ along $\gamma$ with $J\left(s_{1}\right)=0$ and $J\left(s_{2}\right)=0$.

Although a chord, since it has minimum length, may in general have its endpoints conjugate to each other, no two interior points may be conjugate, by Jacobi's theorem. The case when a chord $\gamma_{0}:\left[0, a_{0}\right] \rightarrow \bar{\Omega}$ has conjugate endpoints is therefore somewhat special, and we have called such a chord degenerate.

Lemma 4.1 Suppose that $\partial \Omega$ has positive second fundamental form. Let $\gamma:[0, a] \rightarrow$ $\bar{\Omega}$ be a geodesic with $\gamma(0) \in \partial \Omega$. Then $\gamma$ meets $\partial \Omega$ transversally at $\gamma(0)$.

Proof. Let $\bar{\Omega}$ be extended as a Riemannian manifold to an open manifold $M$, and extend $\gamma$ as a geodesic to a longer interval $[-b, d]$, for some positive $b, d$. If $\gamma^{\prime}(0)$ were tangent to $\partial \Omega$, since the second fundamental form of $\partial \Omega$ is positive, $\gamma(s)$ could only lie outside $\bar{\Omega}$, to second order as $s \rightarrow 0$. This would contradict the assumption that $\gamma([0, a]) \in \bar{\Omega}$.

Q.E.D.

Our next result is essentially a consequence of the Gauss Lemma.

Lemma 4.2 Suppose $\partial \Omega$ has positive second fundamental form. Let $\gamma:\left[0, a_{0}\right] \rightarrow \bar{\Omega}$ be a nondegenerate chord with unit speed, and write $p_{0}=\gamma(0), w_{0}=\gamma^{\prime}(0)$. For $(p, w) \in S(\bar{\Omega})$, write

$$
\gamma_{w}(s):=\exp _{p}(s w)
$$

Then there is a neighborhood $\mathcal{V}$ of $\left(p_{0}, w_{0}\right)$ in $S(\bar{\Omega}) \cap \pi^{-1}(\partial \Omega)$ such that for all $(p, w) \in \mathcal{V}, w$ points into $\Omega$, and the maximal segment $\gamma_{w}\left(\left[0, a_{w}\right]\right)$ lying in $\bar{\Omega}$ has minimum length among those curves in $\bar{\Omega}$ connecting $p=\gamma_{w}(0)$ to $\gamma_{w}\left(a_{w}\right)$. In particular, for all $(p, w) \in \mathcal{V}, \gamma_{w}$ is a chord. Moreover, $a_{w} \rightarrow a_{w_{0}}=a_{0}$ as $(p, w) \rightarrow$ $\left(p_{0}, w_{0}\right)$.

Proof. Recall that, since $\gamma$ is a chord, $\gamma:[0, a] \rightarrow \bar{\Omega}$ is an embedding. Since $\gamma$ is a nondegenerate chord, there are no conjugate points to $p_{0}=\gamma(0)$ along $\gamma((0, a])$. Let $\bar{\Omega}$ be extended as a Riemannian manifold to an open manifold $M$, and extend $\gamma$ as a geodesic to a longer interval $\left[-b_{0}, d_{0}\right]$ on which $\gamma:\left[-b_{0}, d_{0}\right] \rightarrow M$ is an embedding and has no conjugate-point pairs.

Note that $w_{0}$ points into $\partial \Omega$, by Lemma 4.1. If $(p, w) \in S(M) \cap \pi^{-1}(\partial \Omega)$ is sufficiently close to $\left(p_{0}, w_{0}\right)$, the above properties of $\gamma$ will also be valid for $\gamma_{w}$, by the continuous dependence of solutions of the Jacobi equation (4.1) on initial conditions. That is, $w$ points into $\partial \Omega, \gamma_{w}\left(\left[0, a_{w}\right]\right)$ is a geodesic in $\bar{\Omega}$ with endpoints on $\partial \Omega$, and there is a longer interval $\left[-b_{w}, d_{w}\right]$ on which $\gamma_{w}$ is an embedding into 
$M$ and has no conjugate-point pairs. By Lemma 4.1, $a_{w}$ depends continuously on $(p, w) \in S(M) \cap \pi^{-1}(\partial \Omega)$.

Fix $w$ so that $\gamma_{w}$ has the properties just discussed. Write $y:=\gamma_{w}\left(-b_{w}\right) \in M$. Then as $v$ ranges over a sufficiently small neighborhood of $\gamma_{w}{ }^{\prime}\left(-b_{w}\right)$ in the sphere $S_{y}(M)$, we claim that the geodesics $\gamma_{v}\left(\left[0, c_{v}\right]\right)$ sweep out a neighborhood $\mathcal{U}_{w}$ of $\gamma_{w}\left(\left(-b_{w}, d_{w}\right)\right)$ diffeomorphically. Here $\gamma_{v}(0)=y$ and we choose $\gamma_{v}\left(c_{v}\right)$ to be a point in $M$ near $\gamma_{w}\left(d_{w}\right)$ and after $\gamma_{v}$ leaves $\bar{\Omega}$, such that $c_{v}$ is a continous function of $v$ and such that $\gamma_{v}:\left[0, c_{v}\right] \rightarrow M$ is an embedding without conjugate-point pairs. In fact, the absence of conjugate points implies that the radial segment $\left[0, c_{v}\right] v$ in $T_{y}(M)$ lies inside $\mathcal{E}_{y}$. Therefore, $(v, s) \mapsto \gamma_{v}(s)$ is a local diffeomorphism on a neighborhood of $\{w\} \times\left(-b_{w}, d_{w}\right)$, and by suitable restriction, using the embedded property of $\gamma_{w}\left(\left[-b_{w}, d_{w}\right]\right)$, we may obtain a diffeomorphism, as claimed. Let $\delta>0$ be a lower bound for the distance from $\gamma_{w}\left(\left[0, a_{w}\right]\right)$ to the complement of $\mathcal{U}_{w}$ in $M$, for all $(p, w)$ in a neighborhood of $\left(p_{0}, w_{0}\right)$ in $S(M)$.

We may now apply the Gauss Lemma to show that $\gamma_{w}\left(\left[0, a_{w}\right]\right)$ is the unique curve of shortest length inside $\mathcal{U}_{w}$ joining $\gamma_{w}(0)$ to $\gamma_{w}\left(a_{w}\right)$. Specifically, each point $z \in \mathcal{U}_{w}$ may be represented uniquely as $z=\gamma_{v}(s)$ for a unit tangent vector $v$ at $y$ near $\gamma_{w}^{\prime}\left(-b_{w}\right)$. This defines a smooth real-valued function $s=s(z)$ on $\mathcal{U}_{w}$. The Gauss Lemma shows that on $\mathcal{U}_{w}$, the Riemannian gradient of $s(z)$ equals the tangent vector to $\gamma_{v}$, so that any curve $\sigma$ has length at least as large as the change in $s$ along $\sigma$, as long as $\sigma$ stays inside $\mathcal{U}_{w}$. Therefore, any minimizing curve inside $\mathcal{U}_{w}$ must be everywhere transverse or everywhere tangent to the family of geodesics $\left\{\gamma_{v}: v \in S_{y}(M)\right\}$. It follows that $\gamma_{w}$ is the unique curve inside $\mathcal{U}_{w}$ of minimum length between $\gamma_{w}(0)$ and $\gamma_{w}\left(a_{w}\right)$.

It remains to show that, if $(p, w) \in S(M) \cap \pi^{-1}(\partial \Omega)$ is sufficiently close to $\left(p_{0}, w_{0}\right)$, then $\gamma_{w}\left(\left[0, a_{w}\right]\right)$ has shortest length among all curves in $\bar{\Omega}$ joining $\gamma_{w}(0)$ to $\gamma_{w}\left(a_{w}\right)$, that is, that $\gamma_{w}$ is a chord of $\bar{\Omega}$.

Otherwise, there are points $p_{k}$ in $\partial \Omega$ and $\left(p_{k}, w_{k}\right) \in S_{p_{k}}(M)$, with $\left(p_{k}, w_{k}\right) \rightarrow$ $\left(p_{0}, w_{0}\right)$ as $k \rightarrow \infty$, such that $\gamma_{w_{k}}$ is not a chord. This means that for each $k$, there is a unit-speed curve $\sigma_{k}:\left[0, C_{k}\right] \rightarrow \bar{\Omega}$ from $p_{k}=\gamma_{w_{k}}(0)$ to $\gamma_{w_{k}}\left(a_{w_{k}}\right)$ which is shorter than $\gamma_{w_{k}}\left(\left[0, a_{w_{k}}\right]\right)$. Since $\gamma_{w_{k}}$ has shortest length inside the open set $\mathcal{U}_{w_{k}}$, the curve $\sigma_{k}$ must include at least one point $\sigma_{k}\left(s_{k}\right)$ in the complement of $\mathcal{U}_{w_{k}}$ in $\bar{\Omega}$. After passing to a subsequence, we may assume that $s_{k} \rightarrow s_{0}, C_{k} \rightarrow C_{0}$ and $\left(\sigma_{k}\left(s_{k}\right), \sigma_{k}^{\prime}\left(s_{k}\right)\right) \rightarrow(z, v) \in S(\bar{\Omega})$. But for all $k, d\left(\sigma_{k}\left(s_{k}\right), \gamma_{w_{k}}\left(\left[0, a_{w_{k}}\right]\right) \geq \delta\right.$, and hence $d\left(z, \gamma\left(\left[0, a_{0}\right]\right)\right) \geq \delta$.

Define $\sigma(s):=\exp _{z}\left(\left(s-s_{0}\right) v\right), \quad 0 \leq s \leq C_{0}$. Then $\sigma_{k} \rightarrow \sigma$ in the $C^{2}$ norm as $k \rightarrow \infty$, so that $\sigma(0)=p_{0}$ and $\sigma\left(C_{0}\right)=\gamma\left(a_{0}\right)$. By Lemma 4.1, $\sigma$ meets $\partial \Omega$ transversally at $\sigma(0)$ and at $\sigma\left(C_{0}\right)$. We may compute that the lengths $l(\sigma)=\lim _{k} l\left(\sigma_{k}\right) \leq$ 
$\lim _{k} l\left(\gamma_{k}\right)=l(\gamma)$. That is, $\sigma$ is also a chord. The uniqueness of $\gamma$ now implies that $\gamma=\sigma$, which contradicts the fact that $d\left(\sigma\left(s_{0}\right), \gamma\left(\left[0, a_{0}\right]\right)\right) \geq \delta$. This contradiction shows that $\gamma_{w}$ is a chord of $\bar{\Omega}$.

The continuity of $a_{w}$ as a function of $(p, w) \in S(\bar{\Omega})$ follows from Lemma 4.1, as noted above.

Q.E.D.

Remark 4 For another variation on the proof of Lemma 4.2, see Step 3 of Example 5.5 below. In that example, the original chord is degenerate, but nearby geodesics are free of conjugate points and are shown to be chords.

\subsection{The Chord Map, and a Geometric Theorem}

Assume that $\bar{\Omega}$ has unique chords. Given $p, q \in \partial \Omega, p \neq q$, and given $0 \leq s \leq d(p, q)$, we define the chord map

$$
\Phi(p, q, s):=\left(\gamma(s), \gamma^{\prime}(s)\right) \in S(\bar{\Omega})
$$

where $\gamma:[0, d(p, q)] \rightarrow \bar{\Omega}$ is the unique unit-speed chord joining $p$ to $q$. ( $\Phi$ will also be defined at the diagonal $p=q$, below.)

The domain of definition of $\Phi$, as given above, may be written as $U:=\{(p, q, s) \in$ $\partial \Omega \times \partial \Omega \times \mathbb{R}: p \neq q, 0 \leq s \leq d(p, q)\}$.

Lemma 4.3 Suppose that any two points of $\partial \Omega$ are connected by a unique chord of $\bar{\Omega}$, and that $\partial \Omega$ has positive second fundamental form. Then $\Phi: U \rightarrow S(\bar{\Omega})$ is continuous on $U$.

Proof. Consider sequences $p_{k} \rightarrow p_{0}, q_{k} \rightarrow q_{0}$ and $s_{k} \rightarrow s_{0}$, where $p_{k}, q_{k} \in$ $\partial \Omega, q_{k} \neq p_{k}, 0 \leq s_{k} \leq d\left(p_{k}, q_{k}\right)$. Write $\gamma_{0}:\left[0, d\left(p_{0}, q_{0}\right)\right] \rightarrow \bar{\Omega}$ for the unique unit-speed chord joining $p_{0}$ to $q_{0}$, and write $\gamma_{k}$ for the unique unit-speed chord joining $p_{k}$ to $q_{k}$. Then, by definition, $\Phi\left(p_{k}, q_{k}, s_{k}\right)=\left(\gamma_{k}\left(s_{k}\right), \gamma_{k}^{\prime}\left(s_{k}\right)\right)$ and $\Phi\left(p_{0}, q_{0}, s_{0}\right)=$ $\left(\gamma_{0}\left(s_{0}\right), \gamma_{0}^{\prime}\left(s_{0}\right)\right)$. After passing to a subsequence, we may assume that $\left(\gamma_{k}\left(s_{k}\right), \gamma_{k}^{\prime}\left(s_{k}\right)\right) \rightarrow$ $(x, v)$ in $S(\bar{\Omega})$. Let $\gamma$ be the the geodesic in $\bar{\Omega}$ with initial conditions $\gamma\left(s_{0}\right)=$ $x, \gamma^{\prime}\left(s_{0}\right)=v$ and having the maximal domain of definition (the value $s_{0}$ is determined so that $\gamma$ enters $\bar{\Omega}$ at $s=0$.) By the uniqueness of solutions to the initial-value problem for the geodesic equation, we have $\gamma=\gamma_{0}$. Since the limit is the same for all subsequences, this shows that $\Phi\left(p_{k}, q_{k}, s_{k}\right) \rightarrow \Phi\left(p_{0}, q_{0}, s_{0}\right)$.

Q.E.D.

The domain $U$ is a $(2 n-1)$-dimensional manifold-with-boundary, which is not compact because the diagonal $\{p=q, s=0\}$ of $\partial \Omega \times \partial \Omega \times \mathbb{R}$ has been omitted. We shall define a compactification $\bar{U}$ of $U$, by adding to $U$, in place of each point 
$(p, p, 0), p \in \partial \Omega$, a copy of the $(n-2)$-sphere $S_{p}(M) \cap T_{p}(\partial \Omega)$ of unit tangent vectors to the boundary of $\Omega$ at $p$. We shall also write this $(n-2)$-dimensional sphere as $S_{p}(\partial \Omega) . \bar{U}$ will become a compact topological manifold-with-boundary, possibly with non-smooth boundary. By abuse of notation, we shall write $(p, v, 0)$ for the point of $\bar{U} \backslash U$ corresponding to $(p, v) \in S_{p}(\partial \Omega)$. A sequence $\left(p_{k}, q_{k}, s_{k}\right)$ from $U$ will converge to the point $(p, v, 0)$ in $\bar{U} \backslash U$ if and only if $q_{k} \rightarrow p, s_{k} \rightarrow 0$ and $\left(p_{k}, v_{k}\right) \rightarrow(p, v)$, where $v_{k}$ is the initial tangent vector to the chord from $p_{k}$ to $q_{k}$. Convergence within $\bar{U} \backslash U$ will be equivalent to convergence in $S(\partial \Omega)$. The chord map $\Phi$ may then be extended to all of $\bar{U}$ by defining $\Phi(p, v, 0):=(p, v) \in S_{p}(\partial \Omega)$ for each $(p, v, 0) \in \bar{U} \backslash U$.

Lemma 4.4 Suppose that any two points of $\partial \Omega$ are connected by a unique chord of $\bar{\Omega}$, and that $\partial \Omega$ has positive second fundamental form. Then $\Phi: \bar{U} \rightarrow S(\bar{\Omega})$ is continuous on $\bar{U}$.

Proof. If $\left(p_{k}, q_{k}, s_{k}\right) \rightarrow\left(p_{0}, q_{0}, s_{0}\right), p_{0} \neq q_{0}$, then the conclusion follows from Lemma 4.3. If $\left(p_{k}, v_{k}, 0\right) \in \bar{U} \backslash U$, and if $\left(p_{k}, v_{k}, 0\right) \rightarrow(p, v, 0) \in \bar{U} \backslash U$, then the conclusion is immediate from the definition $\Phi(p, v, 0):=(p, v)$.

Suppose $\left(p_{k}, q_{k}, s_{k}\right) \rightarrow(p, v, 0) \in \bar{U} \backslash U$. Then by definition, $\Phi\left(p_{k}, q_{k}, s_{k}\right)=$ $\left(\gamma_{k}\left(s_{k}\right), \gamma_{k}^{\prime}\left(s_{k}\right)\right)$, where $\gamma_{k}$ is the unit-speed chord from $p_{k}$ to $q_{k}$, while $\Phi(p, v, 0)=$ $(p, v) \in S_{p}(\partial \Omega)$. But $\left(p_{k}, \gamma_{k}^{\prime}(0)\right) \rightarrow(p, v)$ in $S(\bar{\Omega})$, since $\left(p_{k}, q_{k}, s_{k}\right) \rightarrow(p, v, 0)$, and hence $\left(\gamma_{k}\left(s_{k}\right), \gamma_{k}^{\prime}\left(s_{k}\right)\right) \rightarrow(p, v)$. This shows that $\Phi$ is continuous on the compact topological manifold-with-boundary $\bar{U}$.

Q.E.D.

We are ready to prove the following geometric theorem.

Theorem 4.5 Assume that the boundary $\partial \Omega$ of the compact Riemannian manifold-with-boundary $\bar{\Omega}$ has positive second fundamental form. Suppose that any two points of $\partial \Omega$ are connected by a unique chord, which is nondegenerate. Then any interior geodesic segment $\gamma:(b-\varepsilon, b+\varepsilon) \rightarrow \Omega$ may be extended to a geodesic $\gamma:\left[s_{0}, s_{1}\right] \rightarrow \bar{\Omega}$ which is a chord, that is, which realizes the minimum length between two distinct points $\gamma\left(s_{0}\right)$ and $\gamma\left(s_{1}\right)$ in $\partial \Omega$.

Proof. Without loss of generality, we may assume that $\Omega$ is connected; otherwise, we may work in the connected component of $\Omega$ containing $\gamma(b)$. The conclusion is immediate if the dimension $n=1$; we shall assume from now on $n \geq 2$.

We shall show that the image $\Phi(\bar{U})$ of the chord map is both open and closed in $S(\bar{\Omega})$. Since $S(\bar{\Omega})$ is a bundle with fiber $S^{n-1}, n \geq 2$, over the connected space $\bar{\Omega}, S(\bar{\Omega})$ is connected; it will then follow that $\Phi$ is surjective. 
To show that $\Phi(\bar{U})$ is open, first consider any point $\left(p_{0}, q_{0}, s_{0}\right) \in U$ and denote $\left(x_{0}, v_{0}\right):=\Phi\left(p_{0}, q_{0}, s_{0}\right)$. Write $\gamma_{0}$ for the chord from $p_{0}=\gamma_{0}(0)$ to $q_{0}=\gamma_{0}\left(a_{0}\right)$, and let $w_{0}=\gamma_{0}^{\prime}(0)$. Then $\left(x_{0}, v_{0}\right)=\left(\gamma_{0}\left(s_{0}\right), \gamma_{0}^{\prime}\left(s_{0}\right)\right)$, by definition of $\Phi$. By Lemma 4.1, $\gamma_{0}^{\prime}(0)$ cannot be tangent to $\partial \Omega$, and in fact must point into $\Omega$. Meanwhile, from Lemma 4.2 we know that there is a neighborhood $\mathcal{V}$ of $\left(p_{0}, w_{0}\right)$ in $S(\bar{\Omega}) \cap \pi^{-1}(\partial \Omega)$ such that every $(p, w) \in \mathcal{V}$ is the initial datum $\left(\gamma_{w}(0), \gamma_{w}^{\prime}(0)\right)$ for a chord $\gamma_{w}$ : $\left[0, a_{w}\right] \rightarrow \bar{\Omega}$. So in our case, for every $(p, w) \in \mathcal{V},(p, w)=\left(\gamma_{w}(0), \gamma_{w}^{\prime}(0)\right)$ where $\gamma_{w}$ is a chord. Now consider a unit vector $(x, v) \in S(\bar{\Omega})$ close to $\left(x_{0}, v_{0}\right)$. Let $\gamma:[0, a] \rightarrow \bar{\Omega}$ be the unit-speed geodesic with initial conditions $\gamma\left(s_{1}\right)=x, \gamma^{\prime}\left(s_{1}\right)=v$ and having maximal interval of definition $[0, a]$, choosing the value $s_{1}>0$ so that $\gamma$ enters $\bar{\Omega}$ at $s=0$. Note that since $\gamma_{0}^{\prime}(0)$ is not tangent to $\partial \Omega$, if $(x, v)$ is sufficiently close to $\left(x_{0}, v_{0}\right)$, then $\gamma$ must enter $\bar{\Omega}$ nontangentially. Further, $(p, w):=\left(\gamma(0), \gamma^{\prime}(0)\right)$ will be close to $\left(p_{0}, w_{0}\right)$; in particular, we may achieve that $(p, w) \in \mathcal{V}$. But this implies that $\gamma$ is a chord. Therefore $\Phi\left(p, \gamma(a), s_{1}\right)=(x, v)$. But $(x, v)$ was an arbitrary point of $S(\bar{\Omega})$ near $\left(x_{0}, v_{0}\right)$, so this shows that the restriction of $\Phi$ to $U$ is an open mapping.

Next, consider a point $\left(p_{0}, w_{0}, 0\right) \in \bar{U} \backslash U$, and recall that $\Phi\left(p_{0}, w_{0}, 0\right)=\left(p_{0}, w_{0}\right) \in$ $S_{p_{0}}(\partial \Omega)$. Consider any point $(x, v) \in S(\bar{\Omega})$ close to $\left(p_{0}, w_{0}\right)$. If $x \in \partial \Omega$ and the vector $v$ points into $\Omega$, or if $x$ is not a boundary point, we form the geodesic $\gamma:[0, a] \rightarrow \bar{\Omega}$ with $\gamma\left(s_{1}\right)=x, \gamma^{\prime}\left(s_{1}\right)=v, \gamma(0) \in \partial \Omega$. Since $(x, v)$ is close to $\left(p_{0}, w_{0}\right)$, we find that also $(p, w):=\left(\gamma(0), \gamma^{\prime}(0)\right)$ is close to $\left(p_{0}, w_{0}\right)$, and we conclude as before that $(x, v)$ is in the image of $\Phi$. If $x$ is a boundary point and $v$ points out of $\Omega$, we choose $p=x, w=-v$ and the conclusion follows in the same way.

This shows that the image of $\Phi: \bar{U} \rightarrow S(\bar{\Omega})$ is an open subset of $S(\bar{\Omega})$.

In order to show that $\Phi(\bar{U})$ is closed, we may apply Lemma 4.4 and recall that $\bar{U}$ is compact.

Q.E.D.

Remark 5 The reader might observe that under the hypotheses of Theorem 4.5, $\Phi$ in fact maps $\bar{U}$ homeomorphically onto $S(\bar{\Omega})$. This observation implies a necessary topological condition for any manifold $\bar{\Omega}$ satisfying the hypotheses of the theorem.

\subsection{Criteria for Chord Uniqueness}

We shall conclude this section with two criteria which imply certain of the hypotheses of Theorem 1.1. The first is especially appropriate in a situation where observations about $\bar{\Omega}$ can only be made from its boundary:

Proposition 4.6 Assume that $\partial \Omega$ has positive second fundamental form. For fixed $q \in \partial \Omega$, let $\phi: \bar{\Omega} \rightarrow[0, \infty)$ be given by $\phi(x):=d_{\bar{\Omega}}(x, q)^{2}$. (1) If, for each $q \in \partial \Omega$, $\nabla \phi$ is continuous along $\partial \Omega$, then any two points of $\partial \Omega$ are connected by a unique 
chord. (2) If, moreover, for each $q \in \partial \Omega, \nabla \phi$ is continuously differentiable along $\partial \Omega$, then the chords are nondegenerate.

Proof. Note that the distance function $\zeta:=\sqrt{\phi}$ is a solution of the Riemannian Hamilton-Jacobi equation $\langle\nabla \zeta, \nabla \zeta\rangle \equiv 1, \zeta(q)=0$. The unit vector $\nabla \zeta(p)$ is the final tangent vector to a chord from $q$ to $p$.

(1) Of course, in general the first derivatives of the Lipschitz function $\zeta$ need not exist everywhere on $\partial \Omega \backslash\{q\}$. Our assumption here implies that $\nabla \zeta(p)$ does exist at each $p \in \partial \Omega, p \neq q$, and is continuous there. Then the chord from $q$ to $p$ is unique. Namely, if there were two chords $\gamma$ and $\sigma$ from $q$ to $p$, then they would have distinct terminal tangent vectors, by the uniqueness of solutions to the Cauchy problem for the Jacobi equation (4.1). But then the chord from $q$ to a point $x \in \partial \Omega$ near $p$ would jump from a chord near $\gamma$ to a chord near $\sigma$, as $x$ moves past $p$ in the direction of the difference of final tangent vectors $\gamma^{\prime}(\zeta(p))-\sigma^{\prime}(\zeta(p))$, contradicting continuity of $\nabla \zeta(p)$.

(2) Now suppose that for all $q \in \partial \Omega$, the gradient $\nabla \zeta$ is continuously differentiable along $\partial \Omega \backslash\{q\}$. In terms of the chord map $\Phi$, this means that $(p, \nabla \zeta(p))=$ $\Phi(q, p, \zeta(p))$ is continuously differentiable as a function of $p \in \partial \Omega, p \neq q$, for any fixed $q \in \partial \Omega$. But the chord $\gamma$ from $q$ to $p$, since it is a solution of the system (2.2) of ODE's, depends smoothly on its terminal conditions $\left(\gamma(\zeta(p)), \gamma^{\prime}(\zeta(p))\right)=$ $\Phi(q, p, \zeta(p))$. In consequence, its initial values $\left.(\gamma(0)), \gamma^{\prime}(0)\right)=\Phi(q, p, 0) \in S_{q}(\bar{\Omega})$ depend on $p$ in a $C^{1}$ manner. For similar reasons, $\Phi(q, p, \zeta(p))$ depends smoothly on $\Phi(q, p, 0))$. Thus, the correspondence $p \mapsto \Phi(q, p, 0))$ is a $C^{1}$ local diffeomorphism from $\partial \Omega \backslash\{q\}$ to $S_{q}(\bar{\Omega})$.

Consider a chord $\gamma_{0}$ from $q$ to $p_{0} \in \partial \Omega$, and let $\left\{\gamma_{\lambda}:-\varepsilon<\lambda<\varepsilon\right\}$ be a smooth one-parameter family of geodesics starting from $q=\gamma_{\lambda}(0)$, with $\frac{\partial}{\partial \lambda} \gamma_{\lambda}^{\prime}(0) \neq 0$ at $\lambda=0$. Then for small $\lambda$, the geodesic $\gamma_{\lambda}$ is also a chord, from $q$ to a point $p_{\lambda} \in \partial \Omega$. Namely, the $C^{1}$ local diffeomorphism $\left.p \mapsto \Phi(q, p, 0)\right)$ maps a neighborhood of $p_{0}$ in $\partial \Omega$ onto a neighborhood of $\gamma^{\prime}(0)$ in $S_{q}(\bar{\Omega})$, by the inverse function theorem. Recall that $\gamma_{0}$ meets $\partial \Omega$ transversely at $p_{0}=\gamma_{0}\left(\zeta\left(p_{0}\right)\right)$. Since $\Phi(q, \cdot, 0)$ is a $C^{1}$ local diffeomorphism, and since $\frac{\partial}{\partial \lambda} \Phi\left(q, p_{\lambda}, 0\right)=\frac{\partial}{\partial \lambda} \gamma_{\lambda}^{\prime}(0) \neq 0$ at $\lambda=0$, it follows that $\frac{\partial}{\partial \lambda} p_{\lambda} \neq 0$ at $\lambda=0$. Now any normal Jacobi field $J$ along $\gamma_{0}$, with $J(0)=0$, arises from a one-parameter family $\left\{\gamma_{\lambda}:-\varepsilon<\lambda<\varepsilon\right\}$, as the variation vector field $J(s)=\frac{\partial}{\partial \lambda} \gamma_{\lambda}(s)$ at $\lambda=0$. Since $J(0)=0$, we may assume that for all $\lambda, \gamma_{\lambda}(0)=q$. But $J\left(\zeta\left(p_{0}\right)\right)$ is the component orthogonal to $\gamma_{0}^{\prime}\left(\zeta\left(p_{0}\right)\right)$ of $\frac{\partial}{\partial \lambda} p_{\lambda}$ at $\lambda=0$, which is nonzero, using what we have just shown and transversality. Therefore $J(s) \neq 0$ at $s=\zeta\left(p_{0}\right)$, and since $\gamma_{0}$ is a chord, at all $0<s<\zeta\left(p_{0}\right)$ as well. This shows that $\gamma_{0}$ is nondegenerate.

Q.E.D. 
Remark 6 Somewhat surprisingly, it is not true that the continuity of $\nabla \phi$ implies disconjugacy of the chords of $\bar{\Omega}$. That is, the stronger hypothesis of part (2) of Proposition 4.6 is required to imply the stronger conclusion. See Example 5.5 below.

The second, rather different criterion concludes that chords are unique, assuming a condition which implies their nondegeneracy (compare Proposition 5.1 below):

Proposition 4.7 Consider a Riemannian manifold-with-boundary $\bar{\Omega}$, whose boundary has positive second fundamental form. Suppose for all $q \in \partial \Omega$, each geodesic starting from $q$ leaves $\bar{\Omega}$ strictly before any conjugate point along the geodesic. Then $\bar{\Omega}$ has unique chords (which are nondegenerate).

Proof. Let $\bar{\Omega}$ be extended to become the closure of an open subset of an open Riemannian manifold $M$. Consider $p_{0}, q_{0} \in \partial \Omega$, and write $a_{0}=d\left(p_{0}, q_{0}\right)$. Let $\gamma_{0}$ : $\left[0, a_{0}\right] \rightarrow \bar{\Omega}$ be a chord from $q_{0}=\gamma_{0}(0)$ to $p_{0}=\gamma_{0}\left(a_{0}\right)$. Write $w_{0}=\gamma_{0}^{\prime}(0)$. It follows from Lemma 4.1 that $w_{0}$ points into $\Omega$. Since $\gamma_{0}$ is nondegenerate, according to Lemma 4.2 there is a neighborhood $\mathcal{V}$ of $\left(q_{0}, w_{0}\right)$ in $S(\bar{\Omega}) \cap \pi^{-1}(\partial \Omega)$ such that for all $(q, w) \in \mathcal{V}, \gamma_{w}:\left[0, a_{w}\right] \rightarrow \bar{\Omega}$, defined by $\gamma_{w}(s)=\exp _{q}(s w)$, is a chord. Moreover, using Lemma 4.1, $a_{w} \rightarrow a_{0}$ as $(q, w) \rightarrow\left(q_{0}, w_{0}\right)$. Another application of Lemma 4.1 shows that $a_{w} \in(0, \infty)$ is smooth as a function of $(q, w)$. Meanwhile, since $\gamma_{0}=\gamma_{w_{0}}$ is nondegenerate, $\exp _{q_{0}}$ maps a neighborhood $\mathcal{U}$ of $w_{0}$ in $T_{q_{0}} \bar{\Omega}$ diffeomorphically onto a neighborhood of $p_{0}$ in $M$. We may assume that $\mathcal{U}$ is a subset of $\mathcal{V}$. In particular, for $p$ in a neighborhood of $p_{0}$ in $\partial \Omega$, there is a unique $w(p) \in \mathcal{U} \cap S_{q_{0}} M$ such that $\gamma_{w(p)}\left(a_{w(p)}\right)=p$, and $w(p)$ varies smoothly as a function of $p$. Also, $\gamma_{w(p)}$ is a chord, so that $\zeta(p)=a_{w(p)}$. Therefore $\nabla \zeta(p)=\gamma_{w(p)}^{\prime}\left(a_{w(p)}\right)$ is smooth near $p_{0}$. Chord uniqueness now follows from Proposition 4.6.

Q.E.D.

\section{$5 \quad$ Examples}

\subsection{Manifold with a Big Bulge}

Let $\bar{\Omega}$ be a Riemannian manifold-with-boundary, diffeomorphic to the closed ball of $\mathbb{R}^{2}$, which contains in its interior a smooth subdomain $\bar{D}$ isometric to the closed hemisphere of the unit sphere $S^{2} \subset \mathbb{R}^{3}$. We assume that $\partial \Omega$ has positive second fundamental form. Such a manifold may be constructed as a hypersurface of revolution in $\mathbb{R}^{3}$, with a generating curve which begins orthogonally to the axis of revolution with a quarter-circle of radius 1 , and ends at a moment when it is travelling away from the axis of rotation. Alternatively, such an example may be constructed by "pasting in" an isometric copy of the closed hemisphere in the interior of a reference manifold whose boundary has positive second fundamental form. 
We claim that $\bar{\Omega}$ will not have unique chords. Namely, any geodesic $\sigma$ of $\bar{\Omega}$ which enters $D$, crossing $\partial D$ transversally at $x$, will remain inside $D$ for a distance exactly $\pi$, until it crosses $\partial D$ transversally at $y$. The points $x$ and $y$ will be conjugate along $\sigma$. These properties follow from the behavior of geodesics (great circles) of $S^{2}$, or from the Jacobi equation (4.1) above. Also, if $\sigma$ is tangent to $\partial D$ at one point, then it remains forever inside $\partial D$. This implies that no chord of $\bar{\Omega}$ can meet $\bar{D}$.

Observe that the curves in the annulus $\bar{\Omega} \backslash \bar{D}$ joining two points $p, q \in \partial \Omega$ fall into an infinite sequence of homotopy classes. Since $\partial D$ is a geodesic, there is a curve of minimum length in each of these homotopy classes. Now if $q$ remains fixed while $p$ moves along $\partial \Omega$ and a point $\hat{p}$ over $p$ moves along the universal covering space of $\partial \Omega$, these minimum lengths will vary continuously; each will assume the value 0 once, at a moment when $\hat{p}$ is one of the sequence of points over $q$, while all the others will be positive. It follows that for a specific choice of $p \neq q$, two of these minimum lengths for homotopy classes will coincide and provide the minimum length among all homotopy classes. Meanwhile, we have seen that all chords must lie in $\bar{\Omega} \backslash \bar{D}$. Thus, there will be at least two chords joining $q$ to $p$.

The nonuniqueness of chords also follows from Theorem 4.5 above, assuming the chords are nondegenerate. For if $\bar{\Omega}$ had unique and nondegenerate chords, then every interior geodesic would be part of a chord; but a geodesic segment inside $D$ cannot be a segment of a chord, as we have seen above. Alternatively, one may form a simpler argument from the existence of closed geodesics in the boundary of $D$. These closed geodesics also show that the wave equation (1.1) on $\bar{\Omega}$ is not controllable from $\partial \Omega$ (cf. Remark 2.)

The manifold $\bar{\Omega}$ also does not allow any convex functions, as follows from Proposition 5.3 below using a domain slightly larger than $D$.

In the context of boundary control of hyperbolic equations, a similar example was introduced and discussed in detail by Yao ([17]).

\subsection{Manifold with a Bulge of Moderate Size}

Let $\bar{\Omega}$ be a spherical cap, of intrinsic radius $R<\frac{\pi}{2}$, in the unit $n$-sphere $S^{n}$. Then $\partial \Omega$ has positive second fundamental form. Also, $\bar{\Omega}$ has unique chords, which are nondegenerate and have lengths $\leq 2 R$, so that Theorem 1.1 may be applied to show that the spherical wave equation (1.1) may be controlled from the boundary in any time $T>T_{0}=2 R$. Note that the requirement $R<\frac{\pi}{2}$ is sharp, since the normal curvatures of $\partial \Omega$ equal $\cot R$, which becomes negative for $R>\frac{\pi}{2}$.

A second method for proving controllability in the recent literature has been to show that certain Carleman estimates hold, relying on the quantitative properties of a strictly convex function on $\bar{\Omega}$. A real-valued function $v$ on $\bar{\Omega}$ is said to be convex if 
$\nabla^{2} v(X, X) \geq 0$ for all tangent vectors $X$. It is strictly convex if $\nabla^{2} v(X, X)>0$ for all $X \neq 0$. For example, Lasiecka-Triggiani-Yao [8] show that boundary control in any time $>T_{1}$ is possible, provided there is a uniformly convex function $v: \bar{\Omega} \rightarrow \mathbb{R}$, with $T_{1}:=2 \frac{\max |\nabla v|}{c_{0}}$, where the uniform convexity of $v$ is quantified by the minimum value $c_{0}>0$ of the Hessian $\nabla^{2} v(X, X)$ over unit tangent vectors $X$ to $\bar{\Omega}$. Note that, with certain other hypotheses strengthened, Yao ([17]) requires only a coercive vector field, which need not be a gradient, rather than a convex function. The paper [17] introduced the use of geometric methods of Bochner type to the control of hyperbolic P.D.E. See also Tataru's paper [14]. In order to apply the method of [8], an apparently optimal choice of convex function for the spherical-cap example of this subsection would be $v(x):=-\cos r(x)$, where $r(x)$ is the distance from $x$ to the center $x_{0}$. Since $\nabla^{2} v(X, X) \equiv-v<X, X>$ (see [3], p. 173), and since $|\nabla r| \equiv 1$, we compute $c_{0}=\cos R$ and $T_{1}=2 \tan R$. But this estimate blows up as $R \rightarrow \frac{\pi}{2}$, so that the requirement $R<\frac{\pi}{2}$ is again seen to be sharp. We have not found a way to improve on the control time $T_{1}$ using Yao's result with an appropriate coercive vector field. By comparison, the time of control which follows from Theorem 1.1 in this case is $T_{0}=2 R<2 \tan R=T_{1}$.

A far more general class of examples may be given, with analogous properties:

Proposition 5.1 Let $\bar{\Omega}$ be a smooth, compact subdomain of a Riemannian manifold $M$, whose sectional curvatures at $x \in M$ are bounded above by $f(r(x))$, where $r(x)=$ $d\left(x, x_{0}\right)$, for some $x_{0} \in \Omega$. Assume that $\exp _{x_{0}}$, the exponential map of $M$ at $x_{0}$, is defined and injective on the closed ball $\bar{B}_{R}(0) \subset T_{x_{0}} M$, and that $r(x) \leq R$ on $\bar{\Omega}$. We assume that $f:[0, R] \rightarrow \mathbb{R}$ satisfies: (1) $f$ is monotone decreasing; (2) the solution $u_{1}$ of the O.D.E. $u^{\prime \prime}+f u=0$ with initial conditions $u_{1}(0)=1, u_{1}^{\prime}(0)=0$ remains positive on $[0, R]$; and (3) the solution $u_{2}$ of the same O.D.E. with initial conditions $u_{2}(0)=0, u_{2}^{\prime}(0)=1$ has positive first derivative on $[0, R]$. Suppose also that $\partial \Omega$ has positive second fundamental form. Then $\bar{\Omega}$ has unique chords, which are nondegenerate.

Proof. According to Theorem 2 of [6], any geodesic of $\bar{B}_{R}\left(x_{0}\right)=\exp _{x_{0}}\left(\bar{B}_{R}(0)\right)$ has length at most $2 R$ and is free of conjugate points, provided that all hypotheses of this proposition are satisfied, except perhaps the hypothesis of positive second fundamental form. Thus, a unit-speed geodesic $\gamma$ entering $\bar{B}_{R}\left(x_{0}\right)$ at $\gamma(0)$ will leave $\bar{B}_{R}\left(x_{0}\right)$ at $\gamma\left(s_{1}\right)$, where $s_{1} \leq 2 R$, and where $\gamma\left(\left[0, s_{1}\right]\right)$ has no conjugate points. That is, with the Riemannian metric of $M, \bar{B}_{R}\left(x_{0}\right)$ fulfills the hypotheses of Proposition 4.7 , and so must have unique chords. We may now apply Theorem 4.5 to $\bar{B}_{R}\left(x_{0}\right)$, and conclude that any geodesic arc in $\bar{B}_{R}\left(x_{0}\right)$ is the restriction of a chord of $\bar{B}_{R}\left(x_{0}\right)$, and therefore is free of conjugate points. 
In particular, $\bar{\Omega}$ satisfies the hypotheses of Proposition 4.7 , and so has unique chords. Since each chord of $\bar{\Omega}$ is the restriction of a geodesic $\gamma\left(\left[0, s_{1}\right]\right)$ of $M$ without conjugate points, it must be nondegenerate.

Q.E.D.

Remark 7 Consider any compact subdomain $\bar{\Omega}_{1} \subset \Omega$, and any solution $u$ of (1.1) having finite energy. It follows from Remark 3 above and from Tataru's trace theorem [15] that the trace of the conormal derivative of $u$ will be in $L^{2}\left(\partial \Omega_{1} \times(0, T)\right)$. This gives us boundary control for either Neumann or Robin controls in optimal Sobolev spaces.

For example, under the hypotheses of Proposition 5.1, we have boundary control for either Neumann or Robin controls in optimal Sobolev spaces for $\Omega \times(0, T)$.

Corollary 5.2 Suppose that for some radius $R$, the Riemannian manifold-withboundary $\bar{\Omega}$ satisfies the hypotheses of Proposition 5.1. Then the wave equation (1.1) on $\bar{\Omega}$ is controllable from $\partial \Omega$ in any time $T>T_{0}=2 R$.

Proof. In order to compute the optimal time $T_{0}$ for boundary control, according to Theorem 1.1 above, we only need to know an upper bound for the diameter of $\partial \Omega$ in the Riemannian distance of $\bar{\Omega}$. But each chord of $\bar{\Omega}$ is a segment of a geodesic of $\bar{B}_{R}\left(x_{0}\right)$ which has length $\leq 2 R$, as shown in the proof of Proposition 5.1. Hence $T_{0}=\operatorname{diam}_{\bar{\Omega}}(\partial \Omega) \leq 2 R$. Optimality of $T_{0}$ follows from Proposition 3.2. $\quad$ Q.E.D.

The reader will note that Corollary 5.2 may be proved more directly, without referring to Proposition 4.7, since Theorem 2 of [6] implies that any geodesic has length at most $2 R$, and the conclusion follows from Proposition 3.2.

The spherical-cap example just considered is the special case of Proposition 5.1 with sectional curvatures $\equiv f(r) \equiv 1$. In this special case, the precise interval allowed for either of the conditions (2) or (3) of Proposition 5.1 is $R<\frac{\pi}{2}$. When $f(r)$ is not constant, however, these maximum intervals may differ: compare Example 5.4 below.

The reader will notice that if $\bar{\Omega}$ has curvature satisfying the hypotheses of Proposition 5.1, then the function $r(x)$ has convex level sets. This follows from condition (3), along with the Rauch Comparison Theorem in its differentiated form (see [4], pp. 215-217.) In fact, for a tangent vector $V$ to the distance sphere $\partial B_{r_{1}}\left(x_{0}\right)$, the Rauch Comparison Theorem implies that the second fundamental form of $\partial B_{r_{1}}\left(x_{0}\right)$ satisfies the inequality

$$
B(V, V) \geq \frac{u_{2}^{\prime}\left(r_{1}\right)}{u_{2}\left(r_{1}\right)}|V|^{2} .
$$

The presumably optimal choice of convex function is then $v(x):=\psi(r(x))$, where $\psi^{\prime}(r) \equiv u_{2}(r)$. Thus, by the methods of [8], one may prove boundary control in any 
time greater than

$$
T_{1}=\frac{2 u_{2}(R)}{\min \left\{u_{2}^{\prime}(r): 0 \leq r \leq R\right\}} .
$$

Again in this general case, it may be seen that $T_{1}>T_{0}=2 R$, unless $f(r) \equiv 0$.

\subsection{Disconjugate Cross Sections, but No Convex Function: the Frisbee}

For a rotationally symmetric Riemannian manifold, we may use the term cross section for a geodesic through the center of symmetry of the manifold. The language is suggested by the special case $n=2$, where this geodesic cuts the manifold into two congruent pieces. This curve would be called a "diameter" in elementary geometry, a term we shall avoid, since $\operatorname{diam}_{\bar{\Omega}}(\partial \Omega)$ may be substantially less than the length of a cross section in a general rotationally symmetric manifold, and in the example we are about to present in particular.

Example 5.1, and the first part of Example 5.2, above, deal with rotationally symmetric manifolds of constant sectional curvature one. We saw in that situation that three properties are lost simultaneously as the radius $R$ increases beyond the critical radius $\frac{\pi}{2}$ !: chord uniqueness, the existence of a convex function and disconjugacy (the absence of any pair of conjugate points) of the cross sections. The first two properties are major hypotheses in theorems about boundary controllability of (1.1) (see Theorem 1.1 and [8].) The third property is intuitively related to disconjugacy of all geodesics which start at the boundary, which implies the first property, assuming that the boundary has positive second fundamental form (Proposition 4.7 above.)

In order to compare the various hypotheses of Theorem 1.1 above with the hypothesis of the existence of a convex function whose gradient is outward along $\partial \Omega$, as required by [8], for example, we shall consider an example with nonconstant sectional curvatures. We shall construct a rotationally symmetric manifold whose cross sections are locally length-minimizing, but which does not support a convex function. Chords will not be unique. This example is a large flat $n$-disk surrounded by a moderate-sized region of positive curvatures and a thin region of negative curvature, resembling the inside surface of the flying toy known as the Frisbee (apologies to the Wham-O Corporation.)

Let $\bar{\Omega}$ be diffeomorphic to the ball $B^{n}$ and radially symmetric. Then the Riemannian metric may be given in spherical coordinates by $d s^{2}=d r^{2}+u(r)^{2} d \theta^{2}$, where $d \theta^{2}$ denotes the Riemannian metric of the unit $(n-1)$-sphere. The parameter $r=r(x)$ is then the distance from $x \in \bar{\Omega}$ to the center $x_{0}$. The sectional curvatures of $\bar{\Omega}$ at $x$ will lie between $K_{1}(r)$ and $K_{2}(r)$, where $u$ satisfies the Jacobi 
equation $\frac{d^{2} u}{d r^{2}}+K_{1}(r) u=0$; and where $K_{2}(r)=u^{-2}\left(1-\left(\frac{d u}{d r}\right)^{2}\right)$, as may be verified using the Gauss equations for the hypersurfaces $r=$ const. In the special case $n=2$, of course, there is only one sectional curvature at each point, the Gauss curvature, which equals $K_{1}(r)$.

For our example, we choose $\bar{\Omega}$ to be the closed ball $r \leq R_{2}$ in $\mathbb{R}^{n}$. Let $u(r)=$ $r, 0 \leq r \leq R_{0} ; u(r)=R_{0} \frac{\cos \left(r-r_{0}\right)}{\cos \left(R_{0}-r_{0}\right)}, R_{0}<r \leq R_{1} ;$ and $u(r)=u\left(R_{1}\right) \frac{\cosh \beta\left(r-r_{1}\right)}{\cosh \beta\left(R_{1}-r_{1}\right)}, R_{1}<$ $r \leq R_{2}$. Here $r_{0}, r_{1}, R_{0}<R_{1}<R_{2}$ and $\beta$ are positive constants to be determined. Then $u$ will have a Lipschitz-continuous first derivative provided that $r_{0}$ and $r_{1}$ are chosen so that $-\tan \left(R_{0}-r_{0}\right)=\frac{1}{R_{0}}, \beta \tanh \beta\left(R_{1}-r_{1}\right)=-\tan \left(R_{1}-r_{0}\right),-\pi / 2<$ $R_{0}-r_{0}<0$ and $0<R_{1}-r_{0}<\arctan \beta$. The radial-normal sectional curvatures of $\Omega$, in a distributional sense, are then $K_{1}(r)=0,0 \leq r \leq R_{0} ; K_{1}(r)=1, R_{0}<r \leq R_{1}$; and $K_{1}(r)=-\beta^{2}, R_{1}<r \leq R_{2}$. The boundary of $\Omega$ will have positive second fundamental form provided that $\frac{d u}{d r}\left(R_{2}\right)>0$, that is, $R_{2}>r_{1}$. We require also that the boundary of the ball $D \subset \Omega$ described by the inequality $r<R_{1}$ have negative second fundamental form, that is, $0<R_{1}-r_{0}<\pi / 2$.

To be specific, based on casual examination of a Frisbee, we choose $\beta=6$. For mathematical convenience, we choose $R_{0} \gg 1$, which entails that $r_{0}$ be slightly greater than $R_{0}$. We shall further choose $R_{1}$ so that $\partial D$ has small negative normal curvatures, thus $0<R_{1}-r_{0} \ll 1$, and choose $R_{2}$ to give $\partial \Omega$ small positive normal curvatures: $0<R_{2}-r_{1} \ll 1$.

Then any cross section $\sigma$ of $\Omega$, given by $0 \leq r \leq R_{2}$ with two antipodal points of $S^{n-1}$ as spherical coordinate, is a geodesic of $\bar{\Omega}$ without conjugate points. Namely, a Jacobi field $J$ which starts out at the center $x_{0}=\sigma(0)$ with $|J(0)|=1$ and $J^{\prime}(0)=0$ will have length $|J(r)|=1$ for all $0 \leq r \leq R_{0}$, and $|J(r)|=\cos \left(r-R_{0}\right)$ for all $R_{0} \leq r \leq R_{1}$. But $0<R_{1}-R_{0} \ll 1$, which implies that $|J(r)|$ remains close to 1 on the interval $\left[0, R_{1}\right]$. We assume that $\tan \left(R_{1}-R_{0}\right)<\beta$; then $|J(r)|>0$ on the final interval $\left[R_{1}, R_{2}\right]$ as well. Since, for all $r, J^{\prime}(r)$ is a scalar multiple of $J(r)$, the length of any normal Jacobi field $J(r)$ satisfies the scalar Jacobi equation $\frac{d^{2}|J|}{d r^{2}}+K_{1}(r)|J|=0$, and a Sturm theorem shows that $J$ cannot have two zeroes in the interval $\left[-R_{2}, R_{2}\right]$. This implies that a cross section cannot have conjugate points. This also implies that the cross sections of $\bar{\Omega}$ have shortest length in a $C^{0}$ neighborhood.

We claim that there can be no strictly convex function $v: \bar{\Omega} \rightarrow \mathbb{R}$ which has positive normal derivative on $\partial \Omega$. In support of our claim, we shall first show

Proposition 5.3 Suppose a Riemannian manifold $\Omega$ contains a subdomain $\bar{D}$ whose boundary has negative first fundamental form. If $w: \bar{\Omega} \rightarrow \mathbb{R}$ is subharmonic on $D$, the Hessian of $w$ is nonnegative at points of $\partial D$, and $w$ is constant on $\partial D$, then $w$ is constant on $\bar{D}$. 
Proof. We integrate $\Delta w$ over $D$, and find that

$$
\int_{\partial D} \frac{\partial w}{\partial \nu}=\int_{D} \Delta w \geq 0
$$

where $\nu$ is the outward unit normal vector to $\partial D$. In particular, either there is a point $p_{0}$ of $\partial D$ at which $\frac{\partial w}{\partial \nu}$ is positive; or else $w$ is harmonic everywhere in $D$, and hence constant on $\bar{D}$ (since it has constant boundary values.)

Let $X$ be any nonzero tangent vector to $\partial D$ at $p_{0}$. Since $w$ is constant on $\partial D$, we compute that the Hessian $\nabla^{2} w(X, X)=-\frac{\partial w}{\partial \nu} B(X, X)<0$, where $B$ is the second fundamental form of $\partial D$. This contradicts local convexity of $w$ at $p_{0}$. Q.E.D.

Since a convex function is a fortiori subharmonic, our claim follows from Proposition 5.3 by symmetrizing a given strictly convex function $v$ in the rotationallysymmetric manifold $\bar{\Omega}$ constructed above, to form a rotationally invariant, strictly convex function $w: \bar{\Omega} \rightarrow \mathbb{R}$. Recall that $\Omega$ contains the subdomain $D$ described by the inequality $r<R_{1}$, whose boundary has negative second fundamental form. Rotational symmetry implies that $w$ is constant on $\partial D$. Proposition 5.3 now implies that $w$ is constant on $D$, which contradicts the strict convexity of $w$.

In order to investigate chord uniqueness for this Frisbee example, it will be useful to have the following proposition.

Proposition 5.4 Assume that $\partial \Omega$ has positive second fundamental form. Suppose there is a subdomain $\bar{D} \subset \Omega$ such that $\partial D$ has negative second fundamental form. If the dimension $n>2$, assume further that $\bar{\Omega}$ and $\bar{D}$ are rotationally symmetric. Then $\bar{\Omega}$ does not have unique chords.

Proof. We first consider the case $n=2$. Choose a point $p \in \partial \Omega$. Suppose on the contrary that any two points of $\partial \Omega$ are connected by a unique chord. Then, according to Lemma 4.4, the chord map $\Phi: \bar{U} \rightarrow S(\bar{\Omega})$ is continuous. This means in particular that as a second point $q \in \partial \Omega$ moves around the connected component of $p$ in $\partial \Omega$, the chord joining $p$ to $q$ varies continuously, and the initial tangent vector to the chord sweeps out the half-circle of $S_{p}(\bar{\Omega})$ from one tangent vector $v$ to $\partial \Omega$ at $p$ to the other, $-v$. The chords themselves sweep out all of $\bar{\Omega}$ continuously. It follows that there is a first point $q_{1} \neq p$ so that the chord from $p$ to $q_{1}$ meets $\bar{D}$, at a point $x \in \partial D$, where it is tangent to $\partial D$. But $\partial D$ has negative second fundamental form at $x$, implying that this chord would lie inside $D \cup\{x\}$ near $x$. However, $q_{1}$ was the first point such that the chord from $p$ to $q_{1}$ meets $\bar{D}$, which implies that the chord from $p$ to $q_{1}$ lies in $\bar{\Omega} \backslash D$, a contradiction.

For the case $n>2$, we have assumed that $\bar{\Omega}$ and $\bar{D}$ are rotationally symmetric. Suppose, contrary to what we want to show, that $\bar{\Omega}$ has unique chords. Choose 
$p \in \partial \Omega$, and choose a totally geodesic two-dimensional submanifold $\bar{N}$ of $\bar{\Omega}$ which contains $p\left(\bar{N}\right.$ is the image of an appropriate two-dimensional subspace ot $T_{x_{0}}(\Omega)$ under the exponential mapping at the center $x_{0}$ of symmetry.) Then $\bar{N}$ is the fixed-point set of an isometry $f$ of $\bar{\Omega}$ with itself, and is totally geodesic. Since $\bar{D}$ is rotationally symmetric, $N$ meets $\partial D$ orthogonally, implying that $N \cap \bar{D}$ is a subdomain of $N$ whose boundary has negative second fundamental form. For the same reasons, $\partial N=\partial \Omega \cap \bar{N}$ has positive second fundamental form. By the $n=2$ case of this Lemma, which we have already proved, $\bar{N}$, considered as a Riemannian 2-manifold with the metric induced from $\bar{\Omega}$, does not have unique chords.

Now any curve in $\bar{N}$ from $p$ to another point $q \in \partial N$ is also a curve of $\bar{\Omega}$, and thus $d_{\bar{N}}(p, q) \geq d_{\bar{\Omega}}(p, q)$. Meanwhile, any chord of $\bar{\Omega}$ from $p$ to any point $q \in \partial N$ must lie in $\bar{N}$, since otherwise its image under $f$ would be another chord, violating uniqueness. Thus the chord of $\bar{\Omega}$ joining $p$ to $q$ is a chord of $\bar{N}$, and is therefore the unique chord in $\bar{N}$ from $p$ to $q$. But this is a contradiction of the $n=2$ case. Q.E.D.

We may now conclude from Proposition 5.4 that the Frisbee example constructed above does not have unique chords. For $D$ in the hypothesis of Proposition 5.4, we may choose the subdomain described by $r<R_{1}$.

Finally, observe that, for the Frisbee example, the wave equation (1.1) is not controllable from the boundary. In fact, the sphere $\left\{r=r_{0}\right\}$ is totally geodesic as a submanifold of $\Omega$, and therefore any of the sphere's own great circles will be closed geodesics of $\Omega$. Any one of these closed geodesics suffices to make boundary controllability impossible (see Remark 2.)

Remark 8 Although the example constructed in this section is only $C^{1,1}$, i.e. the $g_{i j}$ are only Lipschitz continuous, we may smooth the function $u(r)$ in small neighborhoods of $R_{0}$ and of $R_{1}$, so that $u^{\prime \prime}(r)$ remains monotone in each of the small neighborhoods. This $C^{\infty}$ example will enjoy the same properties we have demonstrated for the original $C^{1,1}$ example. The metric of the $C^{\infty}$ example will be arbitrarily close in the $C^{1}$ norm or in the $C^{1, \alpha}$ norm $(0<\alpha<1)$ to the metric of the original example.

\subsection{Convex Function but Nonunique Chords: the Salt Shaker}

In this rotationally symmetric example, positive sectional curvature $\equiv 1$ is concentrated in a ball $B_{R_{0}}\left(x_{0}\right)$ near the center of $\bar{\Omega}$, while the sectional curvature $K_{1}(r)$ which affects Jacobi fields along a cross section becomes identically zero outside that ball. This allocation of curvatures is opposite to Example 5.3. Moreover, we shall construct the metric so that there is a convex function $v: \bar{\Omega} \rightarrow \mathbb{R}$, although there 
will be conjugate points along the cross sections, and chords will not be unique. Control is possible in a finite time, but the optimal time of control may be much less than $\operatorname{diam}_{\bar{\Omega}}(\partial \Omega)$. The example is a truncated cone, topped off at the smaller end with a spherical cap. This resembles a design for salt shakers which are commonly found in American roadside diners, for example.

Let $\Omega$ be diffeomorphic to the ball $B_{R_{1}}\left(x_{0}\right)$, with the Riemannian metric $d s^{2}=$ $d r^{2}+u(r)^{2} d \theta^{2}$. We choose specifically $u(r)=\sin r, 0 \leq r \leq R_{0}$; and $u(r)=$ $\cos R_{0}\left(r-R_{0}\right)+\sin R_{0}, R_{0}<r \leq R_{1}$. Any choice of $0<R_{0}<\frac{\pi}{2}$ will suffice. We require $R_{1}>R_{0}+\cot R_{0}$ : we claim that this will imply that there are conjugate points along any cross section. The extreme sectional curvatures at $x \in \bar{\Omega}$ are $K_{1}(r)=K_{2}(r)=1,0 \leq r \leq R_{0}$; and $K_{1}(r)=0, K_{2}(r)=\tan ^{2} R_{0}\left(r-R_{0}+\right.$ $\left.\tan R_{0}\right)^{-2}, R_{0}<r \leq R_{1}$. Here $r=r(x)$. There is a Jacobi field $J(s)$ along any cross section $\sigma:\left[-R_{1}, R_{1}\right] \rightarrow \bar{\Omega}$, with length $|J(s)|=u_{1}(|s|)$, where $u_{1}$ is the solution of the scalar Jacobi equation $\frac{d^{2} u_{1}}{d r^{2}}+K_{1}(r) u_{1}=0$ with initial conditions $u_{1}(0)=$ $1, u_{1}^{\prime}(0)=0$. We may compute that $u_{1}\left(r_{1}\right)=0$, where $r_{1}=R_{0}+\cot R_{0}<R_{1}$, so that the points $\sigma\left(-r_{1}\right)$ and $\sigma\left(r_{1}\right)$ are conjugate, as claimed.

It follows that chords of $\bar{\Omega}$ are not unique. Namely, let $p$ and $q$ be two opposite points of $\partial \Omega$, that is, $\left(R_{1}, \pm \theta_{0}\right) \in\left[0, R_{1}\right] \times S^{n-1}$ in spherical coordinates. If the chord joining $p$ and $q$ were unique, then it would necessarily be the cross section $\sigma(s)=\left(|s|, \frac{s}{s} \theta_{0}\right)$ which joins them, since this is the only curve which is symmetric under all reflections of $\bar{\Omega}$ that fix $q$ and $p$. But $\sigma$ has a pair of conjugate points $\sigma\left( \pm r_{1}\right)$ in the open interval $\left(-R_{1}, R_{1}\right)$, and therefore cannot have shortest length.

In particular, Theorem 1.1 above does not apply to this salt-shaker example.

On the other hand, $\bar{\Omega}$ does support a convex function $v$ with $\frac{\partial v}{\partial \nu}>0$ on $\partial \Omega$. For example, the function $v$ may be constructed in the rotationally-symmetric form $v=$ $\psi(r)$, with $\psi(0)=0$ and $\frac{d \psi}{d r}=u(r)$. We may apply the results of Lasiecka-TriggianiYao [8] to obtain boundary control in any time greater than $T_{1}:=2 \frac{\max |\nabla v|}{c_{0}}$, where $c_{0}$ is a positive lower bound on convexity of $v$, as in the second paragraph of subsection 5.2 above. With the convex function $v$ just constructed, we find $c_{0}=\cos R_{0}$ and $\max |\nabla v|=u\left(R_{1}\right)$, so that $T_{1}=2\left(L+\tan R_{0}\right)$. Here we have written $L:=R_{1}-R_{0}$ for convenience.

In order to find an estimate for the optimal time of control $T_{0}$ for this example, we may apply Proposition 3.2: $T_{0}$ is the maximum length of any geodesic $\gamma$ in $\bar{\Omega}$. This requires also some explicit geometrical computations. Note that $\bar{\Omega}$ has no closed geodesics, since $v(\gamma(s))$ is a convex function of $s$. Any unit-speed geodesic $\gamma$ has a unique point where $r(\gamma(s))$ assumes a minimum value, which we write as $\lambda:=r(\gamma(0))$. Since $\bar{\Omega}$ is rotationally symmetric, $\gamma$ lies in a totally geodesic twodimensional submanifold. Therefore, to compute the length of $\gamma$, we may assume 
with no loss of generality that $\bar{\Omega}$ has dimension $n=2$. Write $\gamma\left( \pm s_{1}\right)$ for the points where $\gamma$ meets $\partial \Omega$. Then the length of $\gamma$ is $2 s_{1}=2 s_{1}(\lambda)$.

If $\lambda \geq R_{0}$, then $\gamma$ lies entirely in the flat subset $A:=\left\{x: R_{0} \leq r(x) \leq R_{1}\right\}$ of $\bar{\Omega}$, and we may compute its length by working in the euclidean annulus $\widetilde{A}:=\{y \in$ $\left.\mathbb{R}^{2}: \tan R_{0} \leq|y| \leq L+\tan R_{0}\right\}$, since $\widetilde{A}$ has the same Riemannian universal cover as $A$. We find that the length of $\gamma$ is maximized if $\lambda=R_{0}$. Therefore in this case, $\gamma$ has length at most $2 \sqrt{L\left(L+2 \tan R_{0}\right)}$, by the Pythagorean theorem. Similarly, the length of any geodesic segment crossing $A$ from one boundary component to the other is at most $\sqrt{L\left(L+2 \tan R_{0}\right)}$.

If $\lambda \leq R_{0}$, then a central segment $\gamma\left(\left[-s_{0}, s_{0}\right]\right)$ of the geodesic is a small circle in the spherical cap $\left\{x: 0 \leq r(x) \leq R_{0}\right\}$. Its length $2 s_{0}$ is less than or equal to $2 R_{0}$. This leads to the rough estimate $T_{0} \leq 2 R_{0}+2 \sqrt{L\left(L+2 \tan R_{0}\right)}$. This estimate has the same asymptotic behavior as $T_{1}$ in the limit as $L \rightarrow \infty$, for fixed $R_{0}<\frac{\pi}{2}$. However, in the approach $R_{0} \rightarrow \frac{\pi}{2}$ to the non-controllable geometry, this estimate is substantially better than $T_{1}$.

Note that for fixed $L$, if $R_{0}$ is close to $\frac{\pi}{2}$, then the conclusion $T_{0}=\operatorname{diam}_{\bar{\Omega}}(\partial \Omega)$ of Theorem 1.1 may easily fail. Namely, if the inner radius $\tan R_{0}$ of the Euclidean annulus $\widetilde{A}$ is large enough, then $\operatorname{diam}_{\bar{\Omega}}(\partial \Omega)$ will be the length of a chord of the outer circle, which has radius $L+\tan R_{0}$, subtending an arc of length $\pi\left[\sin R_{0}+L \cos R_{0}\right]$. As $R_{0} \rightarrow \frac{\pi}{2}$, this chord length approaches $\pi$, which is much less than the length $2 \sqrt{L\left(L+2 \tan R_{0}\right)}$ of the geodesic tangent to the circle $\left\{r=R_{0}\right\}$, which is a lower bound for the optimal time of control $T_{0}$.

This example also serves as an illustration for Proposition 5.1 above, with $f(r) \equiv$ $1,0 \leq r \leq R_{0}$; and $f(r) \equiv 0, R_{0}<r \leq R_{1}$. Condition (3) of that proposition holds on any interval $[0, R], R<\infty$, and in fact $u_{2}=u$ has positive derivative on $[0, \infty)$. However, condition (2) of Proposition 5.1 fails when $R \geq r_{1}$ (recall that we required $R_{1}>r_{1}:=R_{0}+\cot R_{0}$.)

Remark 8 above applies to this example, as well as to Example 5.3, showing that there are $C^{\infty}$ manifolds, in every $C^{1}$ neighborhood of the salt-shaker example, enjoying the same properties.

\subsection{Nonsmooth $\nabla \zeta$ : the Last Bite of the Bagel}

In this last example, we shall contrast the hypotheses of the two parts of Proposition 4.6 , by constructing an example where the gradient $\nabla \zeta$ of the distance function $\zeta$ from a boundary point $q$ is continuous but not $C^{1}$, and where one of the chords is degenerate. In this two-dimensional example, chords are not unique (since the manifold is not simply connected, there are minimizing curves in each homotopy 
class of curves from $q$ to $p$, whose lengths become equal when $p$ is moved around $\partial \Omega$ ); and, indeed, the wave equation (1.1) is not controllable from the boundary (since there are closed geodesics, $c f$. Remark 2.) The reader may find it interesting to make the further effort to find a similar example, such as a subdomain of $\bar{\Omega}$, with these additional properties. Most of our effort in this example is devoted to identifying the distance function $\zeta$ from a point $q \in \partial \Omega$.

Consider a torus of revolution $M$ in $(x, y, z)$-space, obtained by rotating a circle of radius 1 in the open half-space $\{y>0\}$ of the $(y, z)$-plane about the $z$-axis. At the maximum distance $R>2$ from the $z$-axis, $M$ contains a plane circle $\gamma$ of radius $R$, which is a geodesic in $M$. The closed subdomain $\bar{\Omega} \subset M$ will be the "last bite of the bagel" bounded by two circles (of radius 1 ) in planes containing the $z$-axis, chosen so that the distance $\ell=L(0)$ between them, measured along $\gamma$, equals $\pi \sqrt{R}$. Then, in light of the Jacobi equation (4.1), and since the Gauss curvature equals $\frac{1}{R}$ at points of $\gamma$, there is a Jacobi field along $\gamma$ which has zeroes $\left\{q, p_{0}\right\}$ at the point $q$ where it enters $\bar{\Omega}$ and at the point $p_{0}$ where it leaves $\bar{\Omega}$. That is, $p_{0}$ and $q$ are conjugate points along $\gamma$. Write $\sigma_{0}$ for the segment of $\gamma$ from $q$ to $p_{0}$.

Our next aim is to prove, in three steps, that $\sigma_{0}$ is one of a foliating family of chords $\sigma_{\mu}$ of $\bar{\Omega}$ starting at $q$. Let $\bar{\Omega}$ be parameterized by

$$
X(\phi, \theta)=\left(\begin{array}{c}
w(\phi) \cos \theta \\
w(\phi) \sin \theta \\
\sin \phi
\end{array}\right)
$$

where $w(\phi)=R-1+\cos \phi,-\pi \leq \phi \leq \pi, 0 \leq \theta \leq \theta_{0}:=\frac{\pi}{\sqrt{R}}$. Then the unit-speed geodesic arc $\sigma_{0}(s)=X\left(0, \frac{s}{R}\right)$. Recall Clairaut's relation for a geodesic $\sigma$ on a surface of revolution:

$$
w(\phi(s)) \sin \beta(s) \equiv \text { const. }
$$

where $w(\phi(s))$ is, as above, the distance in $\mathbb{R}^{3}$ from $\sigma(s)$ to the $z$-axis, and where $\beta(s)$ is the signed angle from the tangent vector $\sigma^{\prime}(s)$ to the generating curve $\{\theta=$ const.\} through $\sigma(s)$ (see [4].) In particular, since $w(\phi)>0$, if $\beta(0) \in(0, \pi)$ then for all $s$, we have $\beta(s) \in(0, \pi)$.

Step 1: We shall show that $\sigma_{0}$ is one leaf of a foliation $\left\{\gamma_{\lambda}\right\}$ of $\bar{\Omega} \backslash\left\{q, p_{0}\right\}$.

For each $\lambda \in[-\pi, \pi]$, let $\gamma_{\lambda}(s)=X(\phi(s), \theta(s))$ be the unit-speed geodesic of $M$ with the initial conditions $\theta(0)=\frac{\pi}{2 \sqrt{R}}, \theta^{\prime}(0)=1, \phi(0)=\lambda, \phi^{\prime}(0)=0$. Then $\sigma_{0}$ is the restriction of $\gamma_{0}$ to the interval $-\frac{\pi}{2 \sqrt{R}} \leq s \leq \frac{\pi}{2 \sqrt{R}}$, up to reparameterization. Note that each of the circles $\{\phi=$ const. $\in(0, \pi)\}$ has constant positive geodesic curvature in the direction of increasing $\phi$. It follows that for $0<\lambda<\pi$, along $\gamma_{\lambda}, \phi(s)$ reaches a maximum at $s=0$, with maximum value $\phi(0)=\lambda$. Clairaut's relation implies 
that $0<\frac{w(\lambda)}{R} \leq \sin \beta(s)$ everywhere along $\gamma_{\lambda}$. In particular, $\frac{d \theta}{d s}=\frac{\sin \beta}{w(\phi)}$ is uniformly positive, implying that $\gamma_{\lambda}$ will reach $\partial \Omega$ in either direction from $\gamma_{\lambda}(0)$ in a bounded distance.

Let $\nu$ be the unit normal vector to $\gamma_{\lambda}$, pointing in the direction of increasing $\phi$. Then $\nu$ is parallel along $\gamma_{\lambda}$. Since the dimension $n=2$, the Jacobi field $J(s):=$ $\frac{\partial \gamma_{\lambda}}{\partial \lambda}(s)$ along $\gamma_{\lambda}$ may be written as $J(s)=\mathcal{J}(s) \nu(s)$, where $\mathcal{J}(s)$ is a real-valued function. The system of O.D.E.s which form the Jacobi equation (4.1) becomes a single O.D.E.:

$$
\mathcal{J}^{\prime \prime}(s)+K\left(\gamma_{\lambda}(s)\right) \mathcal{J}(s)=0
$$

We claim that the family $\left\{\gamma_{\lambda}: 0 \leq \lambda \leq \pi\right\}$ foliates the upper half $\{0 \leq$ $\phi \leq \pi\}$ of $\bar{\Omega}$, except at $q$ and at $p_{0}$. Our claim is easily verified if $\ell=\pi \sqrt{R}$ is replaced by a small positive value $\ell$, so that $\bar{\Omega}$ is replaced by its subdomain $\bar{\Omega}^{(\ell)}=\left\{X(\phi, \theta):-\pi \leq \phi \leq \pi,\left|\theta-\frac{\pi}{2 \sqrt{R}}\right| \leq \frac{\ell}{2 R}\right\}$. As $\ell$ increases, the first moment when our claim fails would imply that one of the geodesics $\gamma_{\lambda}, 0 \leq \lambda \leq \pi$ has a pair of conjugate points, one on each component of $\partial \Omega^{(\ell)}=\left\{\theta=\frac{\pi}{2 \sqrt{R}} \pm \frac{\ell}{2 R}\right\}$. However, since the family is a foliation for all smaller values of $\ell$, each of the curves $\gamma_{\lambda}$ minimizes the length between its endpoints, by a well-known argument about fields of extremals. But the curve $\{\phi=$ const. $\}$ between the endpoints of $\gamma_{\lambda}$ has length less than the length $\ell$ of $\gamma_{0}$, so that $\gamma_{\lambda}$ must have length $<\ell \leq \pi \sqrt{R}$. At the same time, the Gauss curvature $K=\frac{\cos \phi}{w(\phi)}$ along $\gamma_{\lambda}$ is at most $\frac{1}{R}$, with equality only along $\gamma_{0}$. It follows from the Jacobi equation (5.1) and a Sturm comparison theorem that no conjugate points are possible unless $\lambda=0$ and $\ell=\pi \sqrt{R}$. This proves our claim.

By symmetry, the family $\left\{\gamma_{\lambda}:-\pi \leq \lambda \leq 0\right\}$ foliates the lower half $\{-\pi \leq$ $\phi \leq 0\}$ of $\bar{\Omega} \backslash\left\{q, p_{0}\right\}$. As a result, the entire family $\left\{\gamma_{\lambda}: \lambda \in(-\pi, \pi]\right\}$ foliates all of $\bar{\Omega} \backslash\left\{q, p_{0}\right\}$. This smooth foliation of $\bar{\Omega} \backslash\left\{q, p_{0}\right\}$ extends to a continuous foliation of all of $\bar{\Omega}$. Namely, for $\lambda$ near 0 , the point where $\gamma_{\lambda}$ meets one component of $\partial \Omega$ is a homeomorphism as a function of $\lambda \in \mathbb{R} / 2 \pi$. This follows from the absence of conjugate points along $\gamma_{\lambda} \cap \bar{\Omega}$ for $\lambda \neq 0$. It follows from the field-of-extremals argument that each of the geodesics $\gamma_{\lambda}$, for $-\pi<\lambda \leq \pi$, and $\gamma_{0}=\sigma_{0}$ in particular, is a chord of $\bar{\Omega}$.

Step 2: We shall show that $\sigma_{0}$ is one of a family of geodesics $\sigma_{\mu}$, starting from $q$ and ending at distinct points $p_{\mu} \in \partial \Omega$, and each without conjugate points.

Write $v_{0}:=\sigma_{0}^{\prime}(0) \in T_{q} M$. The family of geodesics $\sigma_{\mu}$ will be defined so that $\sigma_{\mu}$ starts at $q$ with initial direction making an angle $\mu$ with $v_{0}$. Given $\mu \in\left(-\frac{\pi}{2}, \frac{\pi}{2}\right)$, let $\sigma_{\mu}(s):=\exp _{q}\left(s v_{\mu}\right)$, where $v_{\mu}:=(\cos \mu) v_{0}+(\sin \mu) \frac{\partial X}{\partial \phi}(0,0)$. The unit-speed geodesic $\sigma_{\mu}$ enters $\bar{\Omega}$ at $q$ and leaves $\bar{\Omega}$ after a distance $L(\mu)$ at a point near $p_{0}$, 
which we shall write as $p_{\mu}:=\sigma_{\mu}(L(\mu))=: X\left(f(\mu), \theta_{0}\right)$. By the smooth dependence of solutions of (2.2) on initial data, $p_{\mu}$ and $f(\mu)$ will be smooth functions of $\mu$.

We need to show that $f(\mu)$ is an increasing function on a neighborhood of 0 . Note that $\frac{d f}{d \mu}=0$ at $\mu=0$, since the Jacobi field $J(s)=\mathcal{J}(s) \nu(s)=\frac{\partial \sigma_{\mu}}{\partial \mu}(s)$ vanishes at $\mu=0, s=\pi \sqrt{R}$. Here, the real-valued function $\mathcal{J}(s)$ satisfies equation (5.1) along $\sigma_{\mu}$. Since $\mathcal{J}(s)=\sqrt{R} \sin \frac{s}{\sqrt{R}}$ when $\mu=0$, we have $\mathcal{J}^{\prime}=\frac{d \mathcal{J}}{d s}=-1$ at $\mu=0, s=\pi \sqrt{R}$. By continuity, $\mathcal{J}^{\prime}<0$ for $\mu$ close to 0 and for $s$ close to $\pi \sqrt{R}$. As a consequence, $\cos \beta_{\mu}(s)<0$ for all such $\mu$ and $s$. Namely, $\mathcal{J}^{\prime} \nu=J^{\prime}$, which is the covariant derivative $\frac{D}{\partial \mu} \sigma_{\mu}^{\prime}$, and thus

$$
\frac{\partial}{\partial \mu} \cos \beta_{\mu}(s)=\frac{\partial}{\partial \mu}<\sigma_{\mu}^{\prime}(s), \frac{\partial X}{\partial \phi}>=<\frac{D}{\partial \mu} \sigma_{\mu}^{\prime}(s), \frac{\partial X}{\partial \phi}>=\mathcal{J}^{\prime}(s)<\nu, \frac{\partial X}{\partial \phi}>
$$

which is negative. Integrating from $\mu=0$, where $\cos \beta_{0}(s) \equiv 0$, we find that $\cos \beta_{\mu}(s)<0$ for $\mu$ small and positive and for $s$ close to $\pi \sqrt{R}$.

In order to treat quantitative properties of the family $\left\{\sigma_{\mu}\right\}$, we shall compare $M$ to the 2 -sphere $\widetilde{M}$ of radius $\sqrt{R}$, that is, with constant Gauss curvature $\widetilde{K} \equiv \frac{1}{R}$. Choose $\widetilde{q} \in \widetilde{M}$, and identify the tangent space $T_{\widetilde{q}} \widetilde{M}$ with $T_{q} M$ in an isometric way. Write the corresponding family of geodesics in $\widetilde{M}$ as $\widetilde{\sigma}_{\mu}$. Since the family of geodesics (great circles) $\widetilde{\sigma}_{\mu}$ have no conjugate points out to distance $\pi \sqrt{R}$, the same is true of the family $\left\{\sigma_{\mu}\right\}$, by the Rauch Comparison Theorem (see [4], pp. 215-217.)

Now choose $\varepsilon>0$ and write $\theta_{\varepsilon}=\theta_{0}-\varepsilon$ : for $\mu$ in some interval around 0 , by continuity of the family $\left\{\sigma_{\mu}\right\}$, the open geodesic $\operatorname{arcs} \sigma_{\mu}((0, \pi \sqrt{R}))$ cross the circle $\theta=\theta_{\varepsilon}$. For fixed $\varepsilon$, write $s_{\mu}=s_{\mu}^{(\varepsilon)}<\pi \sqrt{R}$ for the unique parameter such that $\sigma_{\mu}\left(s_{\mu}\right)$ lies on the circle $\theta=\theta_{\varepsilon}$. We may estimate the length $s_{\mu}$ of $\sigma_{\mu}\left(\left[0, s_{\mu}\right]\right)$ using the first variation formula for the length of a curve:

$$
\frac{d s_{\mu}}{d \mu}=\left[<V, \sigma_{\mu}^{\prime}>\right]_{0}^{s_{\mu}}+\int_{0}^{s_{\mu}}<V, \sigma_{\mu}^{\prime \prime}>d s
$$

where $V$ is the variation vector field (see [4].) For this variation, $V$ has normal component $\langle V, \nu\rangle=\mathcal{J}$, while its tangential component is chosen so that $V$ remains tangent to the $\phi$-coordinate curves, and in particular, $V\left(s_{\mu}\right)$ is tangent to the curve $\sigma_{\mu}\left(s_{\mu}\right)$ of endpoints. This implies that $\left\langle V(s), \sigma_{\mu}^{\prime}(s)>=\mathcal{J}(s) \cot \beta_{\mu}(s)\right.$. For $\mu$ small and positive, and $\varepsilon$ close to 0 , we have $\mathcal{J}\left(s_{\mu}\right)>0$ and $\cos \beta_{\mu}\left(s_{\mu}\right)<0$, so that $<V, \sigma_{\mu}^{\prime}><0$ at $s=s_{\mu}$. Meanwhile, all other terms in the first variation formula vanish, implying that $\frac{d s_{\mu}}{d \mu}<0$, and hence that $s_{\mu}=s_{\mu}^{(\varepsilon)}<s_{0}^{(\varepsilon)}=R \theta_{\varepsilon}$.

Letting $\varepsilon \rightarrow 0$, we see that $L(\mu)=s_{\mu}^{(0)}<R \theta_{0}=\pi \sqrt{R}$. But the curves $\sigma_{\mu}([0, \pi \sqrt{R}])$ have no conjugate points. This implies in particular that $\frac{d f}{d \mu}(\mu)>0$ for small $\mu>0$, while as we have seen, $\frac{d f}{d \mu}(0)=0$. 
Observe that the symmetry of $\mathbb{R}^{3}$ under the reflection $z \mapsto-z$, corresponding to the symmetry $\phi \mapsto-\phi$ of $\bar{\Omega}$, implies that $f(\mu)$ is an odd function. Thus $f(\mu)$ is strictly increasing on a neighborhood of $\mu=0$.

Step 3: We shall show that each $\sigma_{\mu}$ is a chord of $\bar{\Omega}$, for sufficiently small $\mu$.

By Step 1 above, we know that $\sigma_{0}$ is the unique chord of $\bar{\Omega}$ from $q$ to $p_{0}$. We shall argue, analogously to the proof of Lemma 4.2, to show that for sufficiently small $|\mu|, \sigma_{\mu}$ is the unique chord from $q$ to $p_{\mu}$. Suppose otherwise: then for some sequence $\mu_{k} \rightarrow 0$, there is a unit-speed chord $\tau_{\mu_{k}}:\left[0, b_{k}\right] \rightarrow \bar{\Omega}$ from $q$ to $p_{\mu_{k}}$, distinct from $\sigma_{\mu_{k}}$. As $k \rightarrow \infty$, by compactness of $S(\bar{\Omega})$, a subsequence, still denoted $\tau_{\mu_{k}}$, converges to a geodesic $\tau_{0}:\left[0, b_{0}\right] \rightarrow \bar{\Omega}$ from $q$ to $p_{0}=\tau_{0}\left(b_{0}\right)$. Since, by Clairaut's relation, $\tau_{0}$ is transverse to the circle $\left\{\theta=\theta_{0}\right\}$, we have $b_{0}=\lim _{k \rightarrow \infty} b_{k}$. By continuity of distance, $d\left(q, p_{0}\right)=\lim _{k \rightarrow \infty} d\left(q, p_{\mu_{k}}\right)=\lim _{k \rightarrow \infty} b_{k}=b_{0}$, which shows that $\tau_{0}$ is a chord from $q$ to $p_{0}$. The uniqueness of $\sigma_{0}$ now implies that $\tau_{0}=\sigma_{0}$. As a consequence, the initial tangent vectors $\tau_{\mu_{k}}^{\prime}(0) \rightarrow \tau_{0}^{\prime}(0)=v_{0}$, so for large $k$ there is $\widehat{\mu}_{k}$ close to 0 with $\tau_{\mu_{k}}^{\prime}(0)=v_{\widehat{\mu}_{k}}$, and thus $\tau_{\mu_{k}}=\sigma_{\widehat{\mu}_{k}}$. But $\tau_{\mu_{k}}$ leaves $\bar{\Omega}$ at $p_{\mu_{k}}=X\left(f\left(\mu_{k}\right), \theta_{0}\right)$, while $\sigma_{\widehat{\mu}_{k}}$ leaves $\bar{\Omega}$ at $p_{\widehat{\mu}_{k}}=X\left(f\left(\widehat{\mu}_{k}\right), \theta_{0}\right)$. Since the function $f(\mu)$ is strictly increasing, we see that $\widehat{\mu}_{k}=\mu_{k}$. This shows that $\tau_{\mu}=\sigma_{\mu}$ after all, so that $\sigma_{\mu}$ is the unique chord from $q$ to $p_{\mu}$. In particular, $L(\mu)=d\left(q, p_{\mu}\right)$ for sufficiently small $|\mu|$.

\section{Conclusion:}

As we have seen in Step 2, on a neighborhood of $\mu=0, f$ is a homeomorphism whose inverse $\mu=f^{-1}(\phi)$ has an infinite derivative at $\phi=f(0)=0$. In particular, $f(\mu)=O\left(\mu^{3}\right)$, with formal first differentiation (i.e. $\frac{d f}{d \mu}(\mu)=O\left(\mu^{2}\right)$.) Since for all $\mu$ near $0, \sigma_{\mu}([0, L(\mu)])$ is a chord of $\bar{\Omega}$, we see that the distance function $\zeta$ from $q$ has gradient $\nabla \zeta\left(p_{\mu}\right)=\sigma_{\mu}^{\prime}(L(\mu))$. By the definition of $\beta_{\mu}$, we have

$$
\nabla \zeta\left(p_{\mu}\right)=\cos \beta_{\mu}(L(\mu)) \frac{\partial X}{\partial \phi}\left(f(\mu), \theta_{0}\right)+\frac{\sin \beta_{\mu}}{R}(L(\mu)) \frac{\partial X}{\partial \theta}\left(f(\mu), \theta_{0}\right) .
$$

Next, we apply Clairaut's relation: $w(f(\mu)) \sin \beta_{\mu}(L(\mu))=w(0) \sin \beta_{\mu}(0)=R \cos \mu$. But $w(f(\mu))=R+O\left(f(\mu)^{2}\right)=R+O\left(\mu^{6}\right)$, which leads to $\beta_{\mu}(L(\mu))=\frac{\pi}{2}+\mu+O\left(\mu^{3}\right)$, and thereby $\nabla \zeta\left(p_{\mu}\right)=-\sin \mu \frac{\partial X}{\partial \phi}+\frac{\cos \mu}{R} \frac{\partial X}{\partial \theta}+O\left(\mu^{3}\right)$. To summarize, for $\mu$ near 0 , the coefficients of $\nabla \zeta\left(p_{\mu}\right)$ depend locally diffeomorphically on $\mu$, while $p_{\mu}=X\left(f(\mu), \theta_{0}\right)$ is characterized by the coordinate $\phi=f(\mu)$. Since $f^{-1}$ has an infinite derivative at $\phi=0$, the restriction of $\nabla \zeta$ to $\partial \Omega$ is not differentiable at $p_{0}$. 


\section{References}

[1] Bardos, Claude, Gilles Lebeau and Jeffrey Rauch: Sharp sufficient conditions for the observation, control, and stabilization of waves from the boundary. SIAM J. Control Optim. 30 (1992), 1024-1065.

[2] Chavel, Isaac: Riemannian Geometry, a Modern Introduction. Cambridge University Press, Cambridge 1993.

[3] Choe, Jaigyoung and Robert Gulliver: Isoperimetric inequalities on minimal submanifolds of space forms. Manuscripta Math. 77 (1992), 169-189.

[4] Do Carmo, Manfredo P.: Riemannian Geometry. Birkhäuser, Boston 1992.

[5] Duff, G. F. D.: Partial Differential Equations. University of Toronto Press, 1956.

[6] Gulliver, Robert: On the variety of manifolds without conjugate points. Trans. Amer. Math. Soc. 210, 185-201 (1975).

[7] Lasiecka, Irene, Roberto Triggiani and Peng-Fei Yao: Exact controllability for second-order hyperbolic equations with variable coefficient-principal part and first-order terms. Nonlinear Anal. 30 (1997), 111-122.

[8] Lasiecka, Irene, Roberto Triggiani and Peng-Fei Yao: Inverse/observability estimates for second-order hyperbolic equations with variable coefficients. $J$. Math. Anal. Applications 235 (1999), 13-57.

[9] Lasiecka, Irene, Roberto Triggiani and Peng-Fei Yao: An observability estimate in $L_{2} \times H^{-1}$ for second-order hyperbolic equations with variable coefficients. S. Chen, X. Li, J. Yong, X. Zhou, eds.: Control of Distributed Parameter and Stochastic Systems, Kluwer Academic Publishers, Dordrecht 1999.

[10] Lebeau, Gilles: Contrôle analytique. I. Estimations à priori. Duke Math. J. 68 (1992), 1-30.

[11] Littman, Walter: Near-optimal-time boundary controllability for a class of hyperbolic equations. Control Problems for Systems Described by Partial Differential Equations and Applications (Gainesville, Fla., 1986), 307-312, Lecture Notes in Control and Inform. Sci. 97, Springer, Berlin-New York, 1987. 
[12] Littman, Walter: Remarks on global uniqueness theorems for partial differential equations. These Proceedings.

[13] Ralston, James: Gaussian beams and the propagation of singularities. Studies in Partial Differential Equations (W. Littman, ed.), MAA Studies in Mathematics 23 (1982), 206-248.

[14] Tataru, Daniel: Boundary controllability of conservative PDEs. Appl. Math. Optim. 31 (1995), 257-295.

[15] Tataru, Daniel: On the regularity of boundary traces for the wave equation. Ann. Scuola Norm. Sup. Pisa Cl. Sci. (4) 26 (1998), 185-206.

[16] Taylor, Michael: Tools for P.D.E.: Pseudodifferential Operators, Paradifferential Operators, and Layer Potentials. In press.

[17] Yao, Peng-Fei: On the observability inequalities for exact controllability of wave equations with variable coefficients. SIAM J. Control Optim. 37 (1999), 1568-1599. 